\title{
Non-conservative evolution in Algols: where is the matter? ${ }^{\star}$
}

\author{
R. Deschamps ${ }^{1,2}$, K. Braun ${ }^{2}$, A. Jorissen ${ }^{2}$, L. Siess ${ }^{2}$, M. Baes ${ }^{3}$, and P. Camps ${ }^{3}$ \\ ${ }^{1}$ European Southern Observatory, Alonso de Cordova 3107, 19001 Casilla, Santiago, Chile \\ 2 Institut d'Astronomie et d'Astrophysique, Université Libre de Bruxelles, ULB, CP 226, 1050 Brussels, Belgium \\ e-mail: Romain.Deschamps@ulb.ac.be \\ 3 Sterrenkundig Observatorium, Universiteit Gent, Krijgslaan 281 S9, 9000 Gent, Belgium
}

Received 7 August 2014 / Accepted 15 February 2015

\section{ABSTRACT}

\begin{abstract}
Context. There is indirect evidence of non-conservative evolutions in Algols. However, the systemic mass-loss rate is poorly constrained by observations and generally set as a free parameter in binary-star evolution simulations. Moreover, systemic mass loss may lead to observational signatures that still need to be found.

Aims. Within the "hotspot" ejection mechanism, some of the material that is initially transferred from the companion star via an accretion stream is expelled from the system due to the radiative energy released on the gainer's surface by the impacting material. The objective of this paper is to retrieve observable quantities from this process and to compare them with observations.

Methods. We investigate the impact of the outflowing gas and the possible presence of dust grains on the spectral energy distribution (SED). We used the 1D plasma code CLOUDY and compared the results with the 3D Monte-Carlo radiative transfer code SKIRT for dusty simulations. The circumbinary mass-distribution and binary parameters were computed with state-of-the-art binary calculations done with the BINSTAR evolution code.

Results. The outflowing material reduces the continuum flux level of the stellar SED in the optical and UV. Because of the timedependence of this effect, it may help to distinguish between different ejection mechanisms. If present, dust leads to observable infrared excesses, even with low dust-to-gas ratios, and traces the cold material at large distances from the star. By searching for this dust emission in the WISE catalogue, we found a small number of Algols showing infrared excesses, among which the two rather surprising objects SX Aur and CZ Vel. We find that some binary B[e] stars show the same strong Balmer continuum as we predict with our models. However, direct evidence of systemic mass loss is probably not observable in genuine Algols, since these systems no longer eject mass through the hotspot mechanism. Furthermore, owing to its high velocity, the outflowing material dissipates in a few hundred years. If hot enough, the hotspot may produce highly ionised species, such as Si IV, and observable characteristics that are typical of W Ser systems.

Conclusions. If present, systemic mass loss leads to clear observational imprints. These signatures are not to be found in genuine Algols but in the closely related $\beta$ Lyraes, W Serpentis stars, double periodic variables, symbiotic Algols, and binary B[e] stars. We emphasise the need for further observations of such objects where systemic mass loss is most likely to occur.
\end{abstract}

Key words. binaries: general - radiative transfer - circumstellar matter - methods: numerical

\section{Introduction}

Algol systems are short-period, semi-detached binary systems (with orbital periods from several hours to tens of days) generally composed of a hot (B-A) main-sequence star and a less massive but more evolved companion overfilling its Roche lobe and transferring mass via Roche lobe overflow (RLOF; Kopal 1955; Giuricin et al. 1983).

Although Algol systems have been studied since the 18th century (Goodricke 1783) and seem to be rather simple objects within the zoo of binary systems, it is still unclear whether the mass transfer can be fully conservative or if systemic mass loss is a universal feature of Algols as has already been inferred by Crawford (1955), among others. It has been shown that systemic angular-momentum losses (and thus mass losses) must be involved to reproduce the current state of some observed Algols. For example, Refsdal et al. (1974) found that only a nonconservative evolution can reproduce the system AS Eridani. Massevitch \& Yungelson (1975) noticed that Algol models must lose at least $40 \%$ of their mass in order to reproduce the observed

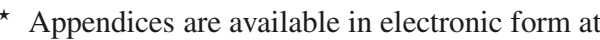
http://www . aanda.org
}

properties. Then, Sarna (1993) concluded that the Algol prototype $\beta$ Per must have lost $15 \%$ of its initial total mass during its evolution and $30 \%$ of its total angular momentum. Statistically, Chaubey (1979) found that semi-detached binaries have lower orbital angular momentum than detached systems of the same total mass. Finally, many other clues (e.g., comparison of grid of models with observations or population synthesis analysis; Mezzetti et al. 1980; Nelson \& Eggleton 2001; van Rensbergen et al. 2011) converge in favour of non-conservative evolution.

Different mechanisms have been proposed to explain systemic mass loss. They may be classified into four groups: bipolar jets (Umana et al. 2000, 2002; Ak et al. 2007; Lomax \& Hoffman 2011), enhanced winds (Chap. 4 of Maeder 2009; Tout \& Pringle 1992), losses through the outer Lagrangian point $\mathcal{L}_{3}$ (Sytov et al. 2007), and "hotspot". The last mechanism appears to be the most promising, because the mass-loss rates derived from the first three mechanisms are too low to account for the inferred systemic mass loss of some Algols. In this scenario, the mass loss is driven by the radiation pressure of a hotspot (van Rensbergen et al. 2008, 2010; Deschamps et al. 2013, hereafter Paper I) that forms when the stream of material coming from the donor via RLOF hits the accreting star and releases energy to heat the 


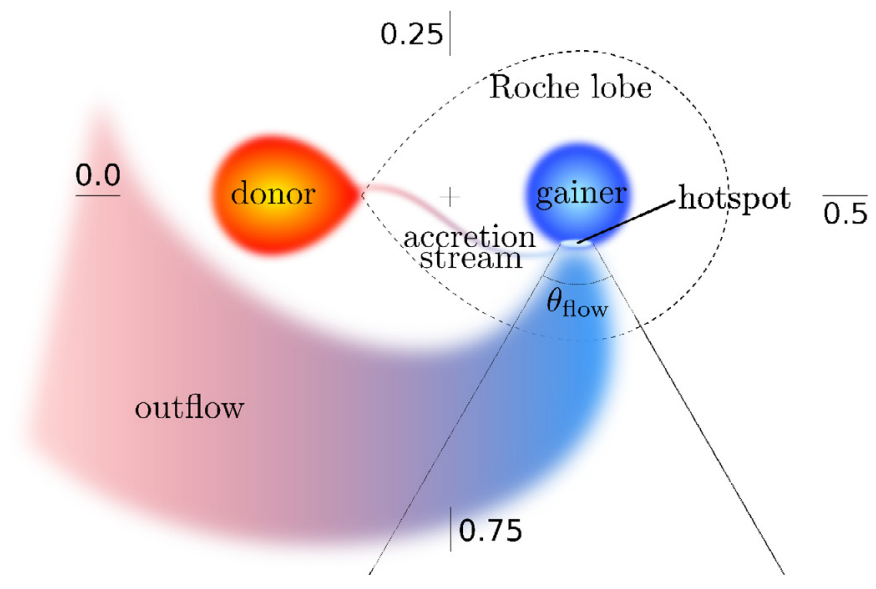

Fig. 1. Schematic overview of an Algol system undergoing systemic mass loss due to a hotspot on the surface of the gainer. The ticks and numbers indicate the different phases (0.0: primary eclipse).

stellar surface and increase the luminosity locally. This hotspot is observed in some Algol-like stars (e.g., Banks et al. 1990; Weiland et al. 1995; Richards \& Albright 1999, in case of direct accretion on the stellar surface and Harmanec 2002; Soydugan et al. 2007 in case of accretion on the edge of a disc).

In the line of van Rensbergen et al. (2008), Deschamps et al. (2013) derived another model for systemic mass loss via hotspot based on energy conservation at the impact location and shows that for a typical initial $3.6 M_{\odot}+6 M_{\odot}$ system with an initial orbital period $P=2 \mathrm{~d} .5$, a large amount of matter (of the order of $1.4 M_{\odot}$, i.e. $15 \%$ of the initial total mass of the system) can be lost during the short-lasting $\left(2 \times 10^{5}\right.$ years $)$, rapid $\left(\dot{M}_{\mathrm{RLOF}}=1 \times 10^{-5}-1 \times 10^{-4} M_{\odot} \mathrm{yr}^{-1}\right)$ mass-transfer phase. This total amount of mass lost is in good agreement with the value inferred by Sarna (1993) to reconcile Algol models with observations. Using the mass-loss history, and assuming that the wind follow the prescription of Castor et al. (1975) for line-driven winds, a density profile for the material surrounding the system was derived.

In this paper, we compare this prediction of systemic mass loss to observations by converting the density profile into observable quantities. We thus analyse light emission and absorption by this material using the codes CLOUDY (Ferland et al. 2013) and SKIRT (Baes et al. 2003, 2011; Camps \& Baes 2015). Section 2 briefly summarises and discusses the assumptions made to obtain the density profile and presents the characteristics of the model system and the set-up of the two codes. The results are presented in Sect. 3. We then compare our findings to Algol observations and broaden our study to promising Algol-related objects in Sect. 4 before concluding in Sect. 5.

\section{The model system}

\subsection{Density profile of the out-flowing matter}

We follow the ballistic trajectory (RLOF-stream) and thus the specific kinetic energy of the material escaping from the donor star via Roche-lobe overflow through the Lagrangian point $\mathcal{L}_{1}$ and accreting onto the gainer star by direct impact (Paper I). When impacting onto the stellar surface, a fraction of this energy heats up the perturbed stellar surface, forming a hotspot (see Fig. 1), and the remainder is radiated away. Mass loss is assumed to occur whenever the total luminosity at the impact location (stellar + hotspot luminosity) exceeds the local Eddington luminosity. The latter is computed using the gainer upper layers' opacity.

We assume that the wind (hereafter outflow) emitted by the hotspot is line-driven and follow the Castor et al. (1975) prescription to compute the outflow speed $v_{\text {flow }}$. Owing to the orbital motion, the outflow will exhibit a spiral shape (Fig. 1; see Sect. 2.2). The acceleration of the particles moving in this spiral outflow will be considerably reduced once the matter leaves the "irradiation" cone defined by the opening angle $\theta_{\text {flow }}$ (whose estimate is provided in Appendix A). It is important to stress that a mass element ejected from the hotspot will escape the system, even if the outflowing material is not constantly lit up by the hotspot, because it exceeds its escape velocity after a small portion of the orbital period ${ }^{1}\left(0.038 \times P_{\text {orb }}\right.$ at the beginning of the mass-transfer phase, $0.011 \times P_{\text {orb }}$ at the end of the non-conservative phase), during which the change of the orientation of the hotspot is negligible.

The density of the material surrounding the system at a given time $t$ and distance $r$ is calculated using the continuity equation (see Paper I for a complete description):

$\rho(r, t)=\frac{\dot{M}_{\text {flow }}(r, t)}{4 \pi f_{\mathrm{c}} r^{2} v_{\text {flow }}(r, t)}$,

where $\dot{M}_{\text {flow }}$ is the systemic mass-loss rate calculated with the BINSTAR code (Siess et al. 2013, Paper I), $4 \pi f_{\mathrm{c}}$ is the solid angle into which the material is ejected, and $f_{\mathrm{c}}$ is the covering factor defined by Eq. (A.9). It is related to the opening angle $\theta_{\text {flow }}$ of the outflow (see Fig. 1). The possibility that the outflowing matter is blocked by the infalling material from the RLOF stream has not been considered, because the hotspot will be elongated as a consequence of stellar rotation. (The accreting star is spun up and no longer synchronised, as observed by Mukherjee et al. 1996.) It is therefore very likely that there is only a small overlap between the stream and the outflow.

By computing the density profile of the circumstellar material at different stages during the evolution, we can estimate the total amount of mass that is potentially detectable. Figure 2 shows the evolution of this quantity during the non-conservative phase. We see that the total amount of detectable mass surrounding the binary is at its maximum before the reversal of the mass ratio (mass ratio $q=1$ at $t \sim 75000 \mathrm{yr}$ : the beginning of the Algol phase). The gainer surface temperature mainly evolves during the rapid phase of mass transfer and reaches a plateau when the mass ratio is reversed.

For our radiative-transfer simulations, we selected three configurations (see Table 1): (i) right after the mass-ratio reversal $(t=80000 \mathrm{yr}$; hereafter model A); (ii) at $t=120000 \mathrm{yr}$ (model B); (iii) at the end of the non-conservative phase $(t=$ $162000 \mathrm{yr}$; model $\mathrm{C}$, our default configuration). In the density profiles of models $\mathrm{A}$ and $\mathrm{B}$, the densities in the ionised region close to the system become so high that an increasing amount of the starlight is transformed into recombination lines and continua that are not explicitly calculated by CLOUDY. Indeed, the model atoms used by CLOUDY account for a finite number of energy levels, and emission from recombinations to higher levels cannot be included in the spectrum. As a result, a significant fraction of the energy released in these recombinations and in the subsequent cascades to the corresponding ground states are missing in the emergent spectrum. In these conditions, it is difficult to predict observable quantities, so we decided to use the density profile of the model $\mathrm{C}$ for the models $\mathrm{A}$ and $\mathrm{B}$

1 As presented in Fig. 3, the escape velocity is reached at a fixed distance from the star, hence after a fixed amount of time. 
R. Deschamps et al.: Non-conservative evolution in Algols: where is the matter?

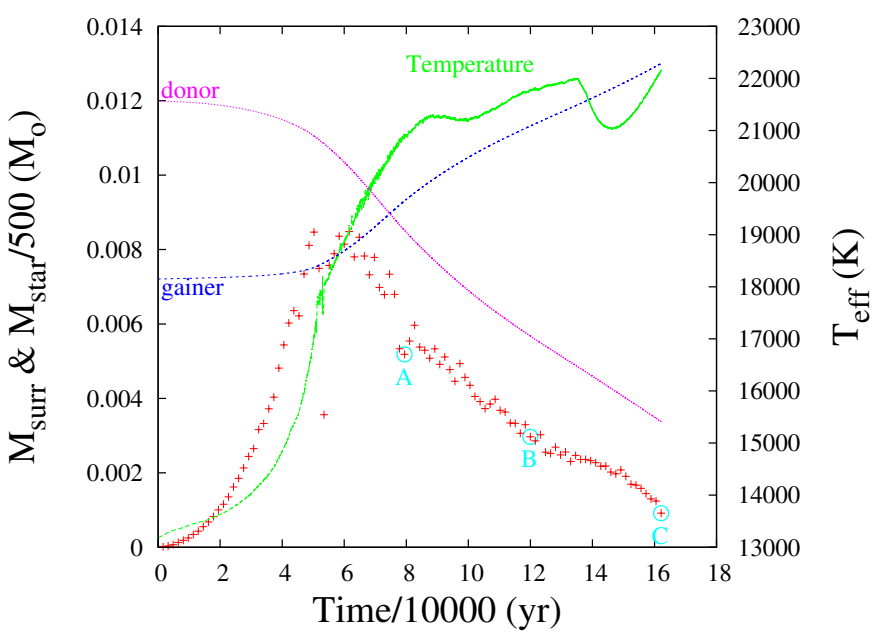

Fig. 2. Red crosses: evolution of the mass surrounding the system (integrated up to the radius where the density of the outflow equals the ISM density; see text). The cyan circles (labelled with the model name) indicate the models selected for the simulations presented in this work. Long-dashed green line (right-hand scale): evolution of the gainer-star surface temperature. Magenta dotted line and dashed blue line: evolution of the masses of the donor and gainer (in unit of $M_{\odot} / 500$ ).

while keeping the hotspot and stellar parameters given by the evolutionary model. Therefore, the simulations corresponding to cases A and B should be considered as exploring the sensitivity of our results to the luminosity of the hotspot, which is the property differing the most between cases $\mathrm{A}, \mathrm{B}$, and $\mathrm{C}$ (Table 1). The sensitivity to the circumstellar shell mass (which was the initial goal for considering cases A, B, and C - but which could not be fulfilled for the reason explained above) - may instead be probed by varying the dust-to-gas ratio $\Phi$. As discussed in Sect. 2.3, a simulation for case $\mathrm{C}$ has been run with 200 times more dust than standard (model labelled "C_hd_0.71" in Table 2). This case thus mimics a situation with a thicker dust shell.

Figure 3 shows the density profile of the outflow for different covering factors $\left(f_{\mathrm{c}}\right)$ for model $\mathrm{C}$. The profile is cut at the distance $r_{\text {cut }}$, where the density drops below the interstellar medium (ISM) value. This occurs at $6.08 \times 10^{17} \mathrm{~cm}(\approx 40600 \mathrm{AU}$ or $2.5 \times 10^{5} A_{\text {orb }}$, where $A_{\text {orb }}$ is the orbital separation) from the gainer for $f_{\mathrm{c}}=0.71$. We do not take any bow-shock formation at this location into account. When the systemic mass loss stops, within 300 years the material disperses and the density profile drops everywhere below the ISM density $\left(\approx 10^{-24} \mathrm{~g} \mathrm{~cm}^{-3}\right)$ because of the very high velocity of the line-driven outflow (Fig. 3).

\subsection{Spiral structure}

Owing to the orbital motion and the fact that the outflow comes from a small area on the star, we must expect a spiral structure to form close to the star. The width of the spiral arm increases as a function of the distance from the star (Fig. 1), and eventually the spiral arm will overlap itself so that the spiral structure fades, giving rise to a disc instead. Using an opening angle for the outflow of $90^{\circ}$ (standard $\theta_{\text {flow }}$ configuration corresponding to $f_{\mathrm{c}}=0.71$ ) and the velocity profile shown in Fig. 3, we estimate the distance at which the spiral fades to be $\approx 3.6 \mathrm{AU}$ $\left(22 A_{\text {orb }}\right)$. This distance is reached by the outflow on the time scale of an orbital period. At greater distances, our assumption of a smooth density profile is thus sufficiently accurate. At shorter distances, we expect deviations due to the spiral structure. In these regions, the density will be the highest when the line of

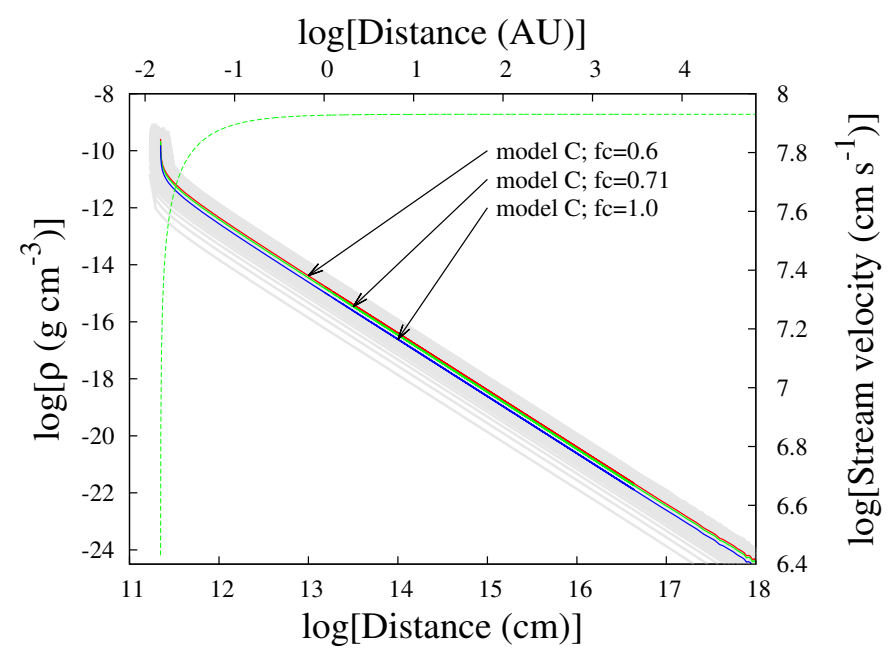

Fig. 3. Density (left-hand scale, solid lines) and velocity (right-hand scale, dashed line) profiles of the outflowing material (similar - but with different parameters - to Fig. 9 of Paper I). Blue line: sphericallysymmetric and isotropic outflow corresponding to a covering factor of $f_{\mathrm{c}}=1$ (see text, Sect. 2.1); green lines: ejection confined in a disc of opening angle $90^{\circ}\left(f_{\mathrm{c}}=0.71\right)$; red line: ejection confined in a disc of opening angle $74^{\circ}\left(f_{\mathrm{c}}=0.6\right)$; The solid light-grey lines are the density profiles for $f_{\mathrm{c}}=0.71$ at 100 different times uniformly distributed during the non-conservative phase.

Table 1. Parameters for the three models; Model A: mass ratio reversal; Model B: intermediate case; Model C: end of non-conservative mass transfer. (For this case, three hotspot temperatures are investigated.)

\begin{tabular}{|c|c|c|c|}
\hline Model A & Gainer & Donor & Hotspot \\
\hline $\begin{array}{l}\text { Radius }\left(R_{\odot}\right) \\
\text { Mass }\left(M_{\odot}\right) \\
\text { Luminosity }\left(L_{\odot}\right) \\
\text { Temperature }(\mathrm{K}) \\
P_{\text {orb }} \\
A_{\text {orb }} \\
\end{array}$ & $\begin{array}{r}3.87 \\
4.65 \\
2496 \\
20840 \\
\end{array}$ & \begin{tabular}{|c|}
5.73 \\
4.26 \\
54 \\
6555 \\
$2.3 \mathrm{~d}$ \\
$15.5 R_{\odot}$ \\
\end{tabular} & $\begin{array}{c}0.14(0.19) \\
- \\
7 \\
35000\end{array}$ \\
\hline Model B & Gainer & Donor & Hotspot \\
\hline $\begin{array}{l}\text { Radius }\left(R_{\odot}\right) \\
\text { Mass }\left(M_{\odot}\right) \\
\text { Luminosity }\left(L_{\odot}\right) \\
\text { Temperature }(\mathrm{K}) \\
P_{\text {orb }} \\
A_{\text {orb }} \\
\end{array}$ & $\begin{array}{c}3.13 \\
5.66 \\
1924 \\
21780\end{array}$ & $\begin{array}{c}6.19 \\
2.83 \\
38 \\
5784 \\
3.3 \mathrm{~d} \\
19.0 R_{\odot} \\
\end{array}$ & $\begin{array}{c}0.24(0.26) \\
- \\
20 \\
35000\end{array}$ \\
\hline Model C & Gainer & Donor & Hotspot \\
\hline Radius $\left(R_{\odot}\right)$ & 3.22 & 9.55 & $\begin{array}{c}0.87 \text { (0.63) (C_xx_0.71_cold) } \\
0.64(0.63) \text { (C_xx_0.71_inter) }) \\
0.49(0.63)(\text { C_xx_0.71_hot })\end{array}$ \\
\hline $\operatorname{Mass}\left(M_{\odot}\right)$ & 6.50 & 1.68 & $-x_{-}-$ \\
\hline Luminosity $\left(L_{\odot}\right)$ & 2195 & 63 & 137 \\
\hline Temperature $(\mathrm{K})$ & 22150 & 5297 & $\begin{array}{c}30000 \text { (C_xx_0.71_cold }) \\
35000 \text { (C_xx_0.71_inter }) \\
40000 \text { (C_xx_0.71_hot })\end{array}$ \\
\hline $\begin{array}{l}P_{\text {orb }} \\
A_{\text {orb }}\end{array}$ & & $\begin{array}{r}8.3 \mathrm{~d} \\
34.9 R_{\odot} \\
\end{array}$ & \\
\hline
\end{tabular}

Notes. The different temperatures of the hotspot correspond to the different simulations labelled (cold, inter, hot). "xx" stands for "ld" or "hd" and designates different dust-to-gas ratios (see text). $P_{\text {orb }}$ and $A_{\text {orb }}$ are the orbital period and separation, respectively. Refer to Table 2 for further explanations. The hotspot radius is calculated using the black body relation (Sect. 2.4.3), and the estimate given by Eq. (3) is shown for comparison in parenthesis. The density profile of model $\mathrm{C}$ is used for all three models (see Sect. 2.1). 
A\&A 577, A55 (2015)

Table 2. Parameters of the simulations performed with the CLOUDY and SKIRT codes.

\begin{tabular}{|c|c|c|c|c|c|c|c|}
\hline Code & \multicolumn{5}{|c|}{ CLOUDY } & \multicolumn{2}{|c|}{ SKIRT } \\
\hline$f_{\mathrm{c}}$ or $\theta_{\text {flow }}$ & \multicolumn{5}{|c|}{$0.60 / 0.71 / 1.00$} & \multicolumn{2}{|c|}{$90^{\circ}$} \\
\hline Density & \multicolumn{2}{|c|}{ Plateau } & \multicolumn{3}{|c|}{ Normal } & \multirow{2}{*}{\multicolumn{2}{|c|}{$\begin{array}{c}\text { Normal } \\
\text { Dust }\end{array}$}} \\
\hline Chemistry & Gas & Gas + Dust & Gas & Gas + I & & & \\
\hline$\Phi$ & - & $2 \times 10^{-5}$ & - & $2 \times 10^{-5}$ & $4 \times 10^{-3}$ & $2 \times 10^{-5}$ & $4 \times 10^{-3}$ \\
\hline Label & C_df_p_0.71 & & $\begin{array}{c}\text { A_df_0.71 } \\
\text { B_df_0.71 } \\
\text { C_df_0.71_cold } \\
\text { C__df_x.xx_inter } \\
\text { C_df_0.71_hot }\end{array}$ & $\begin{array}{c}\text { A_Id_0.71 } \\
\text { B_ld_0.71 } \\
\text { C_ld_0.71_cold } \\
\text { C_ld_x.xx_inter } \\
\text { C_ld_0.71_hot }\end{array}$ & C_hd_0.71 & C_SK_ld & C_SK_hd \\
\hline
\end{tabular}

Notes. "Plateau" (models labelled "p") indicates that the simulation has a constant density in the inner part (see text, Sect. 2.2). $\Phi$ is the dust-to-gas ratio, $f_{\mathrm{c}}$ the covering factor (used for the CLOUDY simulations), and $\theta_{\text {flow }}$ the opening angle (used in the SKIRT simulations to define a 3D dust distribution). In all models, the hotspot temperature is set to $T_{\mathrm{hs}}=35000 \mathrm{~K}$ (i.e., "inter"), except for the models with $f_{\mathrm{c}}=0.71$, for which we applied three different hotspot temperatures ("cold": $T_{\mathrm{hs}}=30000 \mathrm{~K}$; "inter": $T_{\mathrm{hs}}=35000 \mathrm{~K}$; "hot": $T_{\mathrm{hs}}=40000 \mathrm{~K}$ ) with different spectra (see Sect. 2.4.3). "A", "B", and "C" refer to the evolutionary phase (see Fig. 2 and Table 1), "x.xx" is a three-digit number for the covering factor, "df" stands for dust-free, "ld" for low dust (i.e., $\Phi=2 \times 10^{-5}$ ), "hd" for high dust (i.e., $\Phi=4 \times 10^{-3}$ ).

sight points towards the hotspot and will decrease as the orbital motion moves the hotspot out of sight. Ultimately, right before the hotspot points again towards the observer, the density close to the star reaches a minimum because only the material that has been launched orbital periods ago is seen far away from the star.

To mimic this effect and investigate any variability due to the orbital motion, we performed some simulations with a reduced, constant density profile close to the star (in the following, we call these simulations "plateau" and label them with a " $p$ " in the name). Considering the orbital motion $\left(P_{\text {orb }}=8.33\right.$ at the end of the non-conservative phase), we can estimate the density of the outflow ahead of the hotspot. Given the travel time (one orbital period) and the flow velocity, we can estimate the distance travelled by the material, $r_{\mathrm{pl}}$, as well as the density at that location, $\rho_{\mathrm{pl}}$. As a first approximation, we adopt a density profile where the density is equal to $\rho_{\mathrm{pl}}$ up to $r_{\mathrm{pl}}$ and then follow the standard (unmodified) density profile. For the model with $f_{\mathrm{c}}=0.71$, we retrieve a radius of $r_{\mathrm{pl}}=6.05 \times 10^{13} \mathrm{~cm}\left(\approx 4.05 \mathrm{AU}\right.$ or $\left.25 A_{\text {orb }}\right)$ and a corresponding density of $\rho_{\mathrm{pl}}=9.44 \times 10^{-17} \mathrm{~g} \mathrm{~cm}^{-3}$.

\subsection{Chemistry of the outflow: gas composition and dust formation}

One important point for the radiative-transfer analysis is the composition of the gas, which we assume to be that of the donor as listed in Table D.1. The main signature is the depletion of carbon and enhancement of nitrogen during the non-conservative phase (since the mass transfer reveals the inner layers of the giant star).

Although grains can be useful tracers of the cool outermost regions of the outflow, it is not clear whether they can form. Indeed, the escaping material cools as it leaves the gainer's vicinity. Eventually, the temperature drops below the condensation temperature, allowing for the formation of grains. However, $\mathrm{UV}$ and X-ray photons from the hotspot and star (e.g., Ignace et al. 2008) may prevent grain formation. Surprisingly, grains are found in the surroundings of Wolf-Rayet stars (Barniske et al. 2008), which are also emitters of hard radiation.

Since there are no observational constraints on the chemical composition and optical properties of (hypothetical) dust grains around Algol systems, we use the standard ISM model implemented in CLOUDY. However, we exclude carbonaceous grains, because the evolutionary stages of both stars imply $\mathrm{C} / \mathrm{O}<1$ (see Table D.1) so that we can assume that most carbon atoms in the outflow are locked up in $\mathrm{CO}$ molecules. For the slope of the size distribution of the remaining silicate grains, we use the expression suggested by Mathis et al. (1977),

$$
\frac{\mathrm{d} n_{\mathrm{gr}}}{\mathrm{d} a} \propto a^{-3.5}
$$

where $n_{\mathrm{gr}}$ is the number density of grains and $a$ the grain size. The size distribution is discretised in ten bins, which is the maximum number of size bins allowed by Cloudy, ranging from 0.005 to $0.25 \mu \mathrm{m}$, with a ratio $a_{\max } / a_{\min } \approx 1.48$ in each bin, where $a_{\max }$ and $a_{\min }$ are the upper and lower size limits of the bins, respectively. Within each bin, the optical properties of the dust grains are averaged over the respective size interval. For our simulations, the chosen number of bins and their width and location have very little impact on the results. Varying the number and size of the bins only results in a deviation of $\$ 2 \%$ in the flux ratios and magnitudes. Barniske et al. (2008) evaluate the impact of the lower cut-off on the grain size $(0.005 \mu \mathrm{m}$ in our case) and find that only the flux bluewards of $8.3 \mu \mathrm{m}$ is altered, which has no influence on WISE bands 3 and $4(>10 \mu \mathrm{m})$ that will be used in Sect. 4.2.2. Similarly, we ran simulations with different grain sizes and found that the region of the spectra below $\sim 6 \mu \mathrm{m}$ does not depend on grain size so that 2MASS fluxes $(<5 \mu \mathrm{m})$ are not affected.

The optical properties of the grains are calculated by using the Mie theory as described by van Hoof et al. (2004). Because the gainer star has spectral type $\mathrm{B}$, its radiation will probably interfere with the formation of dust grains, and we cannot expect the dust-to-gas ratio $(\Phi)$ to be as high as in the ISM. Instead, we use the results of Barniske et al. (2008), who find dust in the surroundings of WN stars. From their best-fit models, we derive values of $\Phi$ up to $2 \times 10^{-5}$, which we adopt in this work for our standard dusty configuration (models labelled "ld" for lowdust). To investigate the impact of the dust-to-gas ratio, we also computed a model with $\Phi=4 \times 10^{-3}$ (model labelled "hd" for high-dust).

We do not explicitly calculate the destruction of dust grains caused by the high temperatures close to the star. Instead, we use the CLOUDY code to derive a temperature profile for a puregas model (models labelled "df" for dust-free) and then define a dust-free region around the star where the temperature exceeds 
the dust sublimation temperature $(\approx 1400 \mathrm{~K}$ for silicate dust, see Martin \& Rouleau 1991; Laor \& Draine 1993). The circumstellar material will be dust-free up to a transition radius $r_{\text {tr }}$ (radius at which the temperature goes below the condensation temperature).

\subsection{Stellar parameters}

\subsubsection{Luminosities}

The system is composed of the donor star (a late G-type red giant), the gainer star (a B-type dwarf), the hotspot and the material escaping from the hotspot (Fig. 1). The properties of the system (masses, radii, stellar and hotspot luminosities, stellar temperatures; Deschamps et al. 2013) are computed with the BINSTAR code and given in Table 1 for the three selected models.

We can estimate the size of the hotspot $\left(r_{\mathrm{hs}}\right)$ as being identical to the size of the impacting stream $\left(r_{\text {stream }}\right)$, which can in turn be identified with the size of the stream at the Lagrangian point $\mathcal{L}_{1}$. According to Pringle \& Wade (1985), it can be approximated by

$r_{\mathrm{hs}} \sim r_{\mathrm{stream}}=\frac{1}{2} \frac{c_{\mathrm{s}}}{\Omega_{\mathrm{orb}}}$

where $c_{\mathrm{s}}$ is the sound speed of the material leaving the donor star at $\mathcal{L}_{1}$, and $\Omega_{\text {orb }}$ is the orbital angular velocity.

The effective luminosity felt by the material escaping the system depends on its location along the spiral. In the cone with opening $\theta_{\text {flow }}$ above the hotspot (Fig. 1), the escaping material is irradiated by both the gainer and the hotspot ${ }^{2}$. But outside this cone, the hotspot luminosity no longer contributes. We account for this effect by using an effective hotspot luminosity that corresponds to the actual value reduced by a geometrical factor $\gamma$. This geometrical factor is obtained (Appendix B) by assuming that the hotspot luminosity is diluted over a strip-shaped surface encircling the star (blue band in Fig. B.1). The effective flux from that surface reads as

$F_{\text {eff }}=F_{\mathrm{g}}+\gamma F_{\mathrm{hs}}$,

where $F_{\mathrm{g}}=L_{*} / \mathcal{A}_{*}$ and $F_{\mathrm{hs}}=L_{\mathrm{hs}} / \mathcal{A}_{\mathrm{hs}}$ are the surface fluxes of the gainer star and the hotspot, respectively (see Appendix B for details on the calculation of the factor $\gamma$ ). With the quantities given in Table 1 , we find $\gamma \sim 0.05$ for model $\mathrm{C}$.

The use of a time-independent configuration, with an effective flux that constantly irradiates all the surrounding material, is justified by the fact that the cooling time scale (as computed by Cloudy; see Fig. 5) becomes longer than the orbital period at distances $r \approx 3.8 \times 10^{12} \mathrm{~cm}(0.25 \mathrm{au})$ for model C. Owing to the high outflow velocity (up to $900 \mathrm{~km} \mathrm{~s}^{-1}$; Fig. 3), the time needed for the flow to reach that distance is short $\left(\approx 13.93=1.67 P_{\text {orb }}\right.$ at the end of the non-conservative phase). Therefore, the outflow properties (such as temperature or emission spectrum) will not change too much over one orbital period, and several phases of irradiation (as the material passes in front of the hotspot) can be averaged into a constant irradiation.

The spectrum of the gainer star is obtained by interpolating in the "TLUSTY" grid of Lanz \& Hubeny (2007) to match the effective temperature given in the first column of Table 1 . The

\footnotetext{
2 We assume that the donor star does not contribute to the irradiation of the outflowing material, because its luminosity is much lower than that of the gainer (see Table 1). This, however, may not remain true in Algols with a fainter gainer of spectral type A.
}

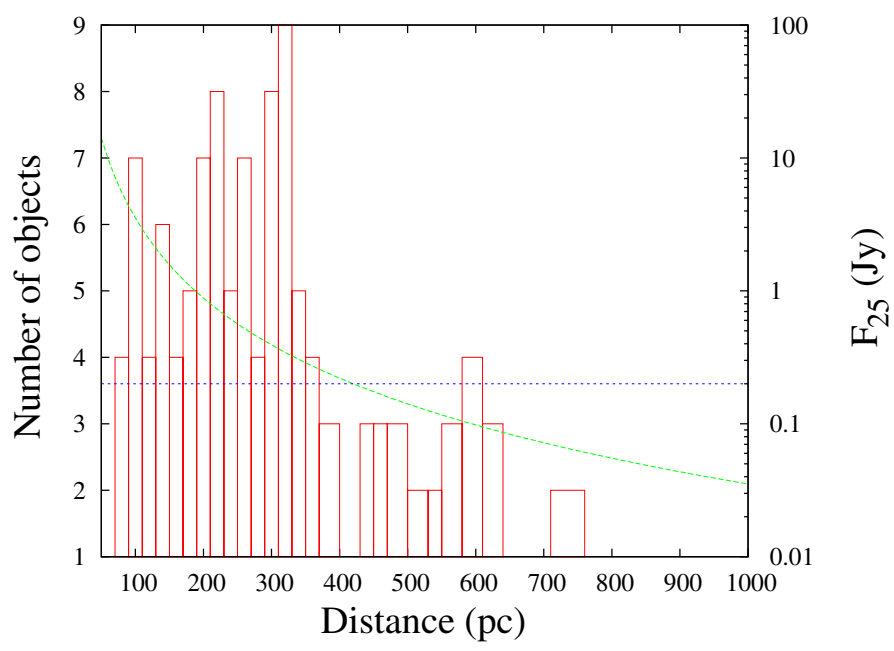

Fig. 4. Distance distribution for observed Algols. The sample of Algols is from Budding et al. (2004). Parallaxes are from the HIPPARcos catalogue (ESA 1997). The dashed green curve represents the flux at $25 \mu \mathrm{m}$ (right-hand scale) as a function of distance for the dusty simulation C_ld_0.71_inter. The flux is integrated over the whole nebula. The dotted blue line is the IRAS $5 \sigma$ limit at $25 \mu \mathrm{m}$. The WISE $5 \sigma$ limit for band $W 4$ is $2600 \mu \mathrm{Jy}$ and is always below the dashed green curve.

donor-star spectrum is taken as a black-body spectrum (see upper panel of Fig. 7). For all the components constituting the total incident spectrum (donor, gainer, hotspot), we use the metallicities listed in Table D.1. The donor has by definition the same abundances as the material ejected, and for the selected models, the gainer star is entirely covered by the donor's material.

\subsubsection{Distance}

To relate absolute fluxes (from the models) with predicted apparent magnitudes for, e.g., the infrared excesses, the distance to the system needs to be fixed. We chose a typical distance from the distribution of measured parallaxes for Algols. In Fig. 4, we present the distance distribution for 152 objects (joint entries in the Budding et al. (2004) and HIPPARCOS (ESA 1997) catalogues), which correspond to about $10 \%$ of all Algols in the Budding et al. (2004) catalogue.

To evaluate which among these systems might be detectable by IRAS, Fig. 4 also presents the $25 \mu \mathrm{m}$ flux of the dusty simulation C_ld_0.71_inter, integrated over the whole nebula, as a function of distance. Only the infrared sources with a flux larger than the IRAS $5 \sigma$ detection limit at $25 \mu \mathrm{m}$ can be detected, so that a system with the same IR emission as our model C_1d_0.71_inter must be within 400 pc of the Sun for its IR excess to be detected, and this includes most of the Algols with a known parallax. Based on this result, a typical distance of $300 \mathrm{pc}$ is adopted to convert model fluxes into apparent fluxes and magnitudes, as listed in Sects. 3 and 4.2.2. To give an idea, $\beta$ Lyrae is located at a distance of $294 \mathrm{pc}$, while W Ser is estimated to lie at 1428 pc (HIPPARCOS catalogue, van Leeuwen 2007). Of course, the apparent fluxes of the model spectral energy distributions (SEDs) can be easily rescaled for comparison with a system at a different distance.

\subsubsection{The temperature of the hotspot}

To assess the dependence of our results on the SED of the hotspot, we used three different hotspot temperatures $T_{\mathrm{hs}}$ in 


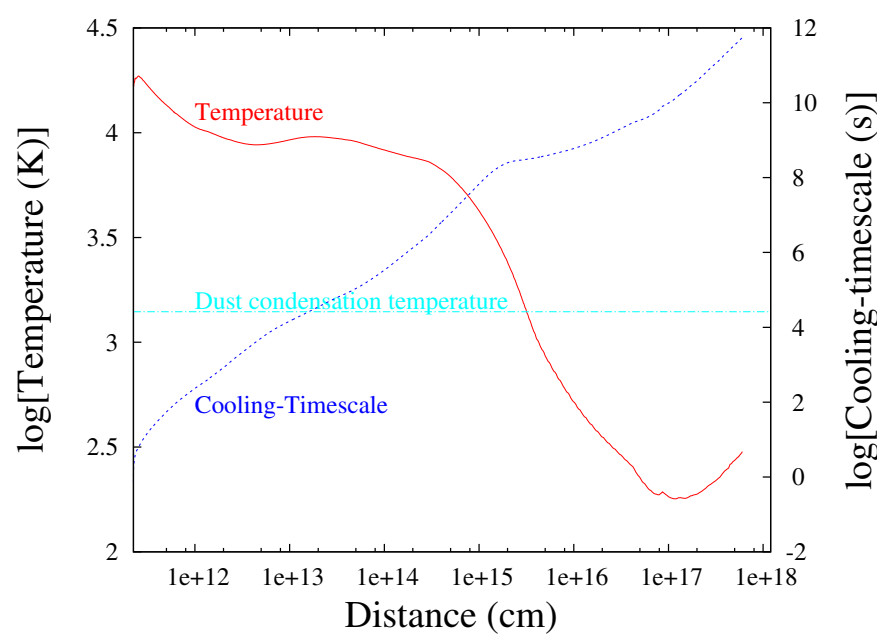

Fig. 5. Temperature profile (solid red line) and cooling time scale (dotted blue line; see text) of the material surrounding the star as a function of distance to the star (model C_df_0.71_inter). The dot-dashed cyan line represents the dust condensation temperature $(1400 \mathrm{~K})$.

model $\mathrm{C}$ with $f_{\mathrm{c}}=0.71$ (see Tables 1 and 2). In the first model (C_xx_0.71_cold), we assumed that the hotspot emits with a B-star spectrum at a temperature of $30000 \mathrm{~K}^{3}$. In the second model (C_xx_0.71_inter), we assumed a temperature of $35000 \mathrm{~K}$ and use an O-star spectrum (also interpolated from the TLUSTY grid). In the third model (C_xx_0.71_hot), we used an O-star spectrum with a temperature of $40000 \mathrm{~K}$. These three temperatures are based on observed hotspot values in Algols with similar properties (stellar masses and orbital periods; van Rensbergen et al. 2011), and the corresponding results are presented in Sect. 3.3. These three models allow the effect of the hotspot properties on the emergent SED to be clearly disentangled.

To satisfy the Stefan-Boltzmann law $L_{\mathrm{hs}}=\sigma T_{\mathrm{hs}}^{4} \mathcal{A}_{\mathrm{hs}}$ (cf. Eq. (B.2)), the radius of the hotspot is treated as a free parameter ( $L_{\mathrm{hs}}$ and $T_{\mathrm{hs}}$ are fixed) and compared to the estimate given by Eq. (3) (see Table 1). Although Eq. (3) can only give a rough approximation of the radius of the hotspot, we see a good match between this value and the one imposed by the Stefan-Boltzmann law.

\subsection{Setup of CLOUDY and SKIRT}

We used version 13.02 of CLOUDY, last described by Ferland et al. (2013), to calculate the ionisation of the gas close to the gainer star and the emission from the dust grains in the outflow at longer distances. CLOUDY calculates the thermal and ionisation balance with a simultaneous treatment of radiative transfer. For the latter, an escape probability formalism is used. In our simulations, the photon energy scale was discretised with a resolution of $\lambda / \Delta \lambda=2000$ down to $\lambda=0.152 \mathrm{~nm}^{4}$. CLOUDY calculates the strengths of $\gtrsim 10^{5}$ spectral lines. We found that the number of energy levels that are used for the Fe II ion can have a strong impact on the resulting temperature profile and thus on the transition radius $r_{\mathrm{tr}}$, and also on the emitted spectrum. Therefore, we used the detailed Fe II model that was developed by Verner et al. (1999) and implemented in CLOUDY. To define

\footnotetext{
3 In our models, the hotspot is a region of the star's atmosphere with a higher temperature, so we use a synthetic star spectrum instead of a black-body spectrum.

$4 \lambda / \Delta \lambda=333$ between CLOUDY's high-energy limit at $12.4 \mathrm{fm}$ and $0.152 \mathrm{~nm}$
}

the spatial step size, Cloudy uses adaptive logic. However, for the models C_df_0.71_hot, C_ld_0.71_hot, C_df_1.00_inter, and C_ld_1.00_inter, we had to define a lower limit for the step size, of the order of $10^{8} \mathrm{~cm}$, to reach convergence.

The ejection of matter triggered by the hotspot is assumed to occur primarily in the orbital plane and is assumed to be seen edge-on. In our spherical 1D simulations with CLOUDY, we take that into account by adjusting the covering factor (equivalent to the factor $f_{\mathrm{c}}$ introduced in Sect. 2.1, Eq. (1)). In Appendix A, we estimate the covering factor (or, equivalently, the opening angle) based on the assumption of isotropic radiation from the hotspot. To study the effect of the choice of opening angle on the results, we performed simulations with different covering factors (the three digits in the label of each simulation: "x.xx").

When assessing the systematic uncertainties due to the 1D geometry and the simplified approach used by CLOUDY for the radiative transfer, we use the SKIRT code, which simulates the general 3D continuum transfer for dust, including absorption, multiple anisotropic scattering, and thermal emission (Baes et al. 2003, 2011; Camps \& Baes 2015). The 3D dust-distribution is represented by a disc that is centred on the gainer star and truncated at its inner edge at the dust condensation radius. The thickness of the disc increases linearly with distance as defined by the opening angle $\theta_{\text {flow }}$ (see Appendix A), and the density inside the disc is assumed to be a function of distance only (see Fig. 3).

The spatial grid consists of 200 concentric shells. The density is set to zero in the directions where the polar angle is outside the range $\pi / 2-\theta_{\text {flow }} / 2 \leq \theta \leq \pi / 2+\theta_{\text {flow }} / 2$, and the extent of the grid cells is adapted accordingly. The positions $r_{i}$ of the grid cells along the radial coordinate follow a power-law distribution in the form

$r_{i+1}=r_{\mathrm{tr}}+\frac{1-R^{i /(N-1)}}{1-R^{N /(N-1)}}\left(r_{\max }-r_{\mathrm{tr}}\right)$,

where $N>i \geq 0$ and $r_{0}=0, r_{\mathrm{tr}}$ is the dust formation radius (212 AU for the model C_ld_0.71_inter; Sect. 3.1), $N=200$ the number of grid points, and $r_{\max }$ is chosen in such a way that the density drops below the ISM density at the outer edge of the grid. Here, $R=800$ is approximately the ratio of the sizes of the outermost and innermost grid cells, $\left(r_{N}-r_{N-1}\right) /\left(r_{2}-r_{1}\right)$.

The functional form of Eq. (5) is motivated by the steeply decreasing density profile. The ratio of the widths of two adjacent grid cells is held constant. This implies that the width of the grid cells increases from the centre towards the outer edge. Therefore, the high-density regions close to the centre are covered by more cells than the outer low-density regions. This allows us to save computing time. The first term on the righthand side of Eq. (5) is introduced because there is a dust-free region around the centre, which does not have to be resolved in the SSKIRT simulations. The composition and size distribution of the dust are the same as in the CCLOUDY models. In the radiative-transfer Monte-Carlo method used in SSKIRT, the radiation field is represented by discrete photon packages. We used $10^{6}$ photon packages per wavelength in our simulations. Our wavelength grid consists of 1000 wavelength points with logarithmic spacing between $1 \mathrm{~nm}$ and $500 \mu \mathrm{m}$, which allows convergence within a reasonable computation time. The different parameters used in the simulations performed with CLOUDY and SKIRT are summarised in Table 2.

\section{Results}

\subsection{Temperature and emissivity profiles}

The first quantity of interest is the outflow-temperature profile, because dust can only form if it falls below the condensation 


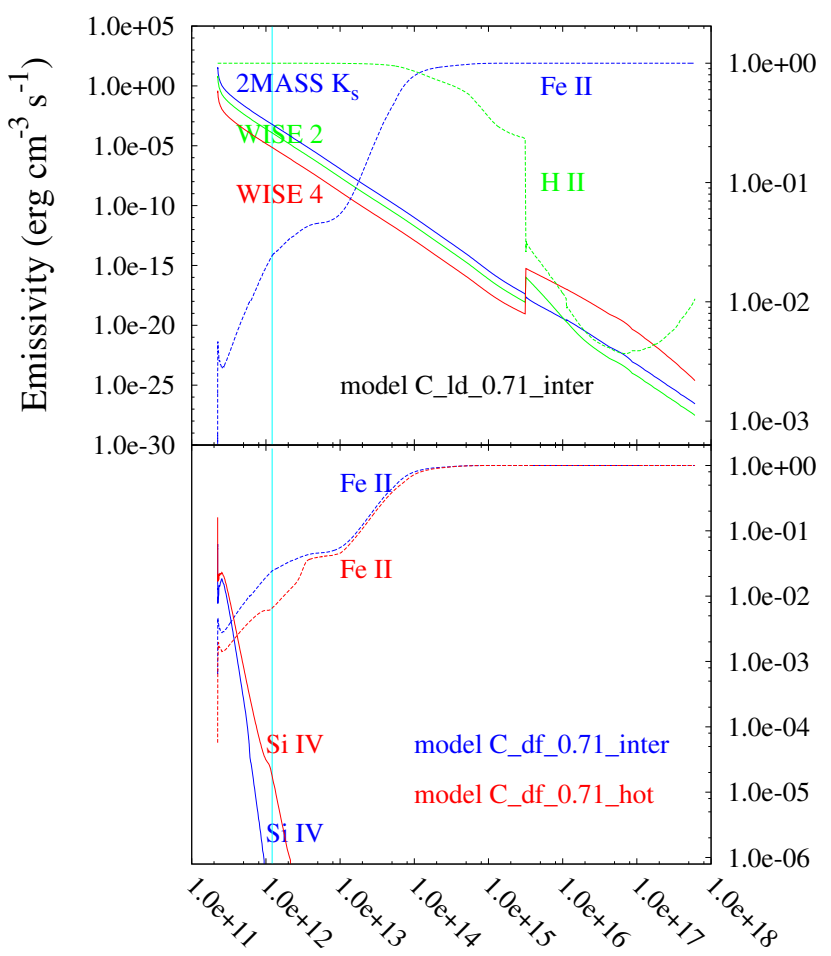

Distance $(\mathrm{cm})$

not the case at smaller radii. In the outer region, the emissivity is low (of the order of $10^{-15} \mathrm{erg} \mathrm{cm}^{-3} \mathrm{~s}^{-1}$ ), but the total emission, which is the integral over the volume, remains large. The infrared excess in the W4 band is therefore visible as a "bump" in the infrared part of the SED from $\sim 10 \mu \mathrm{m}$ towards longer wavelengths (Fig. 9). Owing to the rapidly decreasing density profile, only the dust that is closest to the star contributes to the infrared excess, especially at the lowest wavelengths. For this simulation, as well as for the dust-free one, the inner part is totally ionised (H II, Fe III, and higher). Although Fe II is mainly present in the outer part of the system, the formation of Fe II lines may partially happen close to the star and present some line variability there (see Sect. 3.3 and bottom panel of Fig. 6).

$-\quad$ In contrast, the contribution from Fe II in the outer parts comes . from a smooth, roughly time-steady density distribution and is probably not variable. The resulting observed line will be the sum of the two contributions (variable inner part, steady outer part).

\subsection{Synthetic SEDs}

In this section, we inspect the output spectra in the different regimes, from ultraviolet to infrared, and the impact of the different parameters.

\subsubsection{Optical-UV}

The top panel of Fig. 7 shows the emergent SEDs for the dustfree models C_df_0.60_inter, C_df_0.71_inter, C_df_0.71_cold, and C_df_1.00_inter (the corresponding SEDs for models that contain dust are nearly identical in this spectral region and are therefore not shown). They all show pronounced Balmer and Paschen recombination continua. The Balmer jump in emission leads to observable diagnostics, as further discussed in Sect. 4.3.

In the SEDs of the models C_df_0.71, we notice a slight absorption in the UV up to a wavelength of $\sim 0.2 \mu \mathrm{m}$ (also seen in Fig. 8 for the same model), which is mainly attributable to photoionisation close to the star, where the density is high. This has two consequences: i) this absorption will quickly disappear when the non-conservative phase stops, because the outflowing material will rapidly dissipate (see Sect. 2.1); ii) the UV absorption should show a phase dependence owing to the spiral structure of the outflow. (This prediction is compared to observations of W Ser in Appendix C.)

The bottom panel of Fig. 7 presents the SED for the different models $(\mathrm{A}, \mathrm{B}, \mathrm{C})$ with a covering factor $f_{\mathrm{c}}=0.71$ (corresponding to $\theta_{\text {flow }}=90^{\circ}$ ) and $T_{\mathrm{hs}}=35000 \mathrm{~K}$. Model C exhibits the strongest Balmer and Paschen recombination continua and model A the most prominent UV absorption. As discussed in Sect. 4.3, The presence of the recombination continuum and emission lines and the absence of stellar absorption lines are not typical of Algols, but are a common feature of symbiotic systems or planetary nebulae (Kenyon 1986).

\subsubsection{Far UV-continuum orbital modulation}

The comparison of the results from the model C_df_0.71_inter and the corresponding "plateau" model C_df_p_0.71 (the latter having a lower density in the inner region around the system) allows us to evaluate the impact of the azimuthal dependence of the outflow density, since the line of sight probes different parts of the outflow spiral structure. As discussed in Sect. 2.2, the "plateau" model mimics situations that correspond to orbital phases where the line of sight does not enter the cone of opening $\theta_{\text {flow }}$ (Fig. 1), so when the density close to the star is low. 


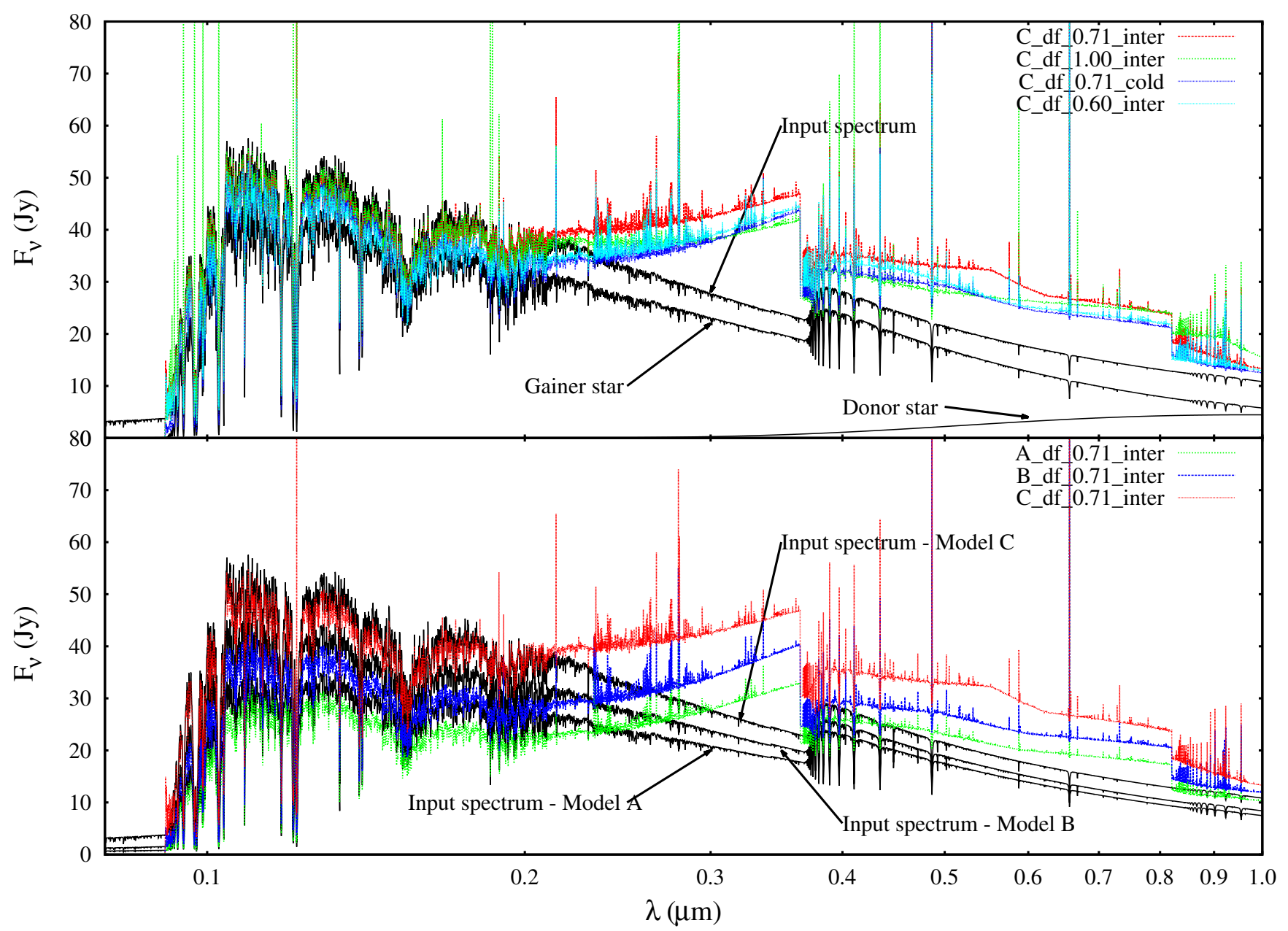

Fig. 7. Top: synthetic SEDs of the dust-free CLOUDY models in the UV-optical regime. The input spectrum is the sum of the spectra of the gainer star, donor star, and hot spot with a temperature of $35000 \mathrm{~K}$ (the last diluted according to the prescriptions of Appendix B). The fluxes are computed for a system distance of 300 pc. Bottom: same as top panel but for the different models A, B, and C (at different evolutionary times). The input spectra are in black from bottom to top (A, B, C).

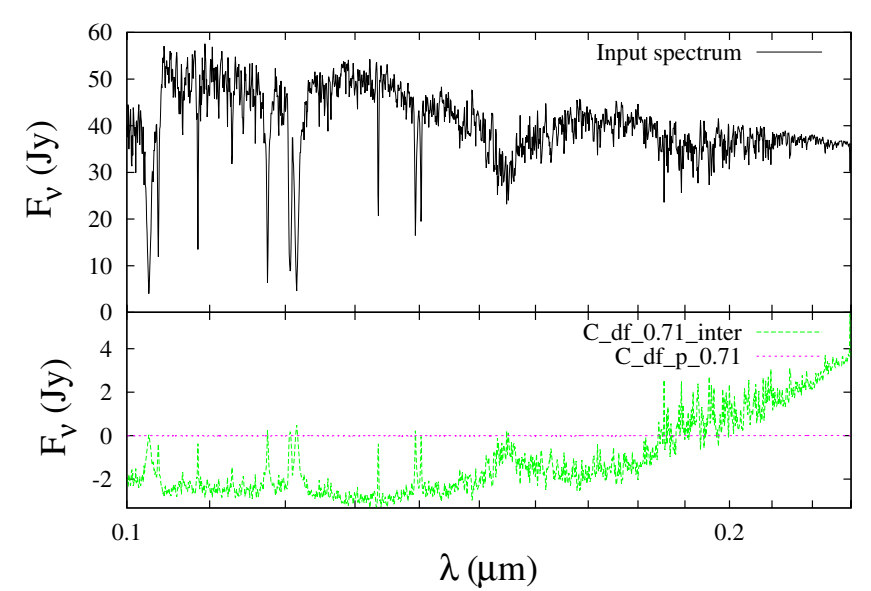

Fig. 8. Synthetic SEDs of dust-free models in the UV regime. The solid black line in the upper panel represents the input stellar spectrum. The lower panel shows the difference between the output and input spectra for the two models identified by the line labels.

Figure 8, which presents the SED in the UV regime for these two models, reveals that the "plateau" model has no far-UV absorption, whereas the non-plateau models have. Thus, one expects an orbital modulation of the far-UV continuum as a consequence of the azimuthal distribution of the outflowing material close to the star. A comparison with far-UV spectro-photometric observations could thus possibly provide insight into the spatial structure of the outflow.

\subsubsection{Emission lines from weakly ionised species}

Figure 7 reveals the presence of many Fe II emission lines for the different models considered, which are best seen in the range $0.2-0.3 \mu \mathrm{m}$ but are also present in the optical. Figure 6 reveals that these lines mainly form in the outer part of the outflow, outside the Roche lobe. Detecting these lines may help to prove that some material is indeed escaping from the binary system. The absence of such lines in the spectra of genuine Algols may thus be considered as an argument against the presence of outflowing matter in these systems.

\subsubsection{Infrared}

Figure 9 shows the characteristic silicate feature that is produced by the dust grains in the outer part of the outflow in our models. All models produce infrared excesses above the $5 \sigma$ IRAS detection limit at 12 and $25 \mu \mathrm{m}$, but only the simulation with the highest dust-to-gas ratio has a $60 \mu \mathrm{m}$ flux detectable by IRAS (for systems located as usual at a distance of $300 \mathrm{pc}$ ). In the "plateau" simulation (C_ld_p_0.71, not shown in the figure), there is no dust emission, because the temperature falls below 
R. Deschamps et al.: Non-conservative evolution in Algols: where is the matter?

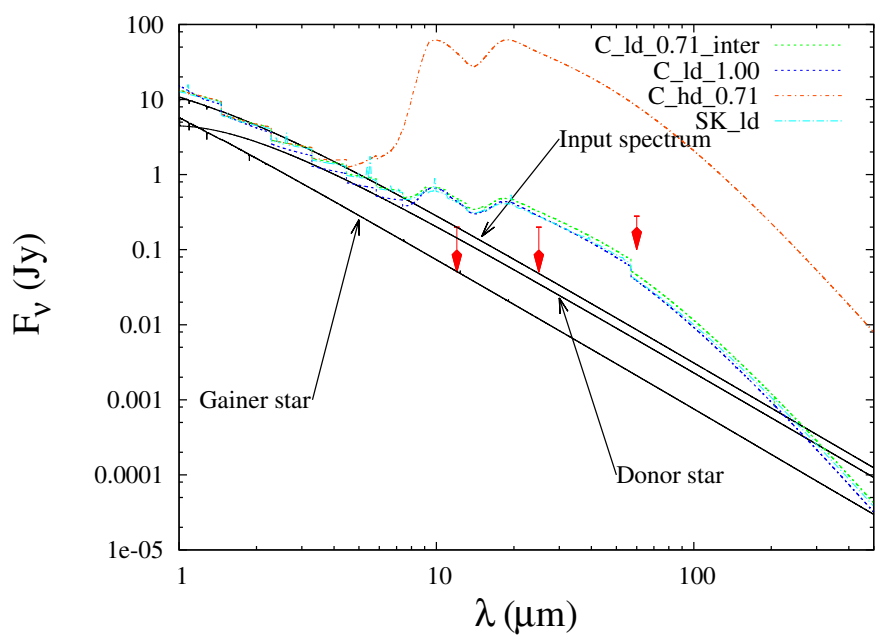

Fig. 9. Same as Fig. 7 but for the IR region, including the SKIRT model C_SK_ld. For clarity, spectral lines have been removed from the CLOUDY SEDs. The red arrows are $5 \sigma$ IRAS upper limits (in the faintsource catalogue): $0.2 \mathrm{Jy}$ for the 12 and $25 \mu \mathrm{m}$ bands and $0.28 \mathrm{Jy}$ for the $60 \mu \mathrm{m}$ band.

the condensation temperature at a location where the density is too low to produce any detectable infrared excesses.

\subsubsection{Synthetic photometry}

To ease the comparison of our models with observations, Table 3 presents, for all our models, the magnitudes in the Strömgren uvby system and the corresponding colour indices, using the passbands and zero points of Bessell (2011) and assuming that the models are located $300 \mathrm{pc}$ away from the Sun. As expected, the systems with a strong Balmer continuum emission appear with a negative value of $c_{1}=(u-v)-(v-b)$. Figure 10 presents our models in the $\left[m_{1,0} ; c_{1,0}\right]$ diagram compared with data from the Long-Term Photometry of Variables project (Manfroid et al. 1991). Only comparison stars (labelled A, B, or C in their catalogue) have been retained to define the main sequence. The $c_{1,0}$ and $m_{1,0}$ indices are not affected by interstellar reddening and are defined by (Strömgren 1966)

$$
\begin{aligned}
c_{1,0} & =c_{1}-0.2(b-y)=[(u-v)-(v-b)]-0.2(b-y) \\
m_{1,0} & =m_{1}+0.18(b-y)=[(v-b)-(b-y)]+0.18(b-y)
\end{aligned}
$$

While our input spectrum falls within the main-sequence population (Strömgren 1966), our models deviate considerably towards lower $c_{1,0}$. Deviations in $m_{1,0}$ are also observed for some models. In addition to the Strömgren photometry, Table 4 shows the SDSS magnitudes and two colour indices, which we calculated using the passbands provided by Moro \& Munari (2000) and the zero points from Fukugita et al. (1996). In Table 5, we also present the 2MASS and WISE band magnitudes that will be used in Sect. 4.2.2. WISE magnitudes are computed according to Tokunaga \& Vacca (2005), based on transmission curves from the Explanatory Supplement to the WISE All-Sky Data Release Products (Cutri et al. 2012) ${ }^{5}$. The 2MASS response curves are set up according to $\mathrm{ADPS}^{6}$ (See also the "Explanatory

\footnotetext{
5 http://wise2.ipac.caltech.edu/docs/release/allsky/ expsup/index.html, 2014-02-27, Sect. IV.4.h, Fig. 5a and Table 3.

6 http://ulisse.pd.astro.it/Astro/ADPS/Systems/Sys_ new_021/index_n021.html, 2014-03-05.
}

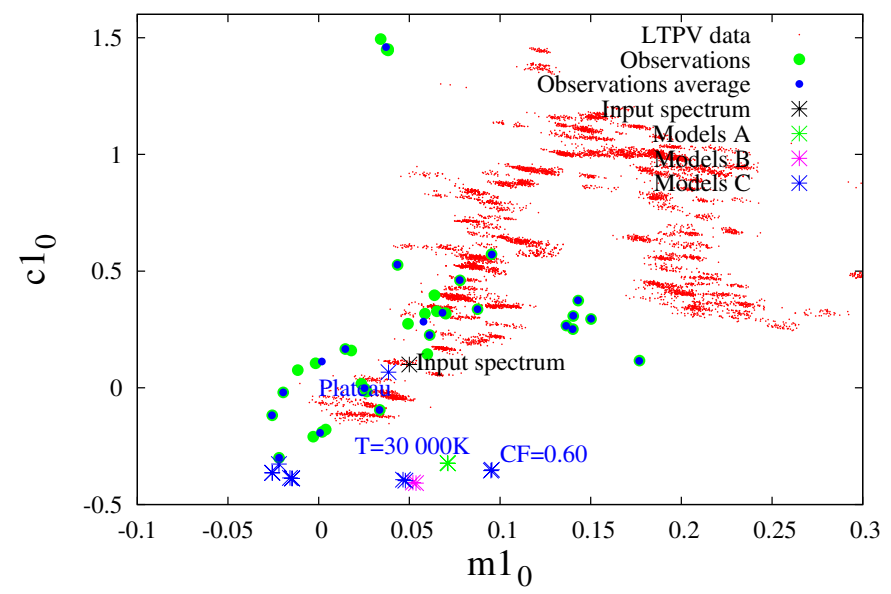

Fig. 10. Strömgren photometry $\left[m 1_{0} ; c 1_{0}\right]$ diagram for our models (crosses) and for comparison, normal main-sequence stars (red dots) from the Long-Term Photometry of Variables project (Manfroid et al. 1991, to be discussed in Sect. 4.3). From the latter, only data obtained with the Danish telescope (system 7) were retained. For clarity, the models are not labelled, and only their general properties are indicated in blue. We refer to Table 3 for the values. We added observations of $\mathrm{W}$ Ser, B[e], and Be stars for comparison (green dots). The blue dots are the average values for the latter objects when several observations are available.

Table 3. Magnitudes in Strömgren (uvby) bands and corresponding colour indices.

\begin{tabular}{lccccccc}
\hline \hline Model & $u$ & $v$ & $b$ & $y$ & $b-y$ & $m_{1}$ & $c_{1}$ \\
\hline A_df_0.71 & 5.08 & 5.31 & 5.22 & 5.20 & 0.02 & 0.07 & -0.32 \\
A_ld_0.71 & 5.09 & 5.31 & 5.22 & 5.20 & 0.02 & 0.07 & -0.32 \\
B_dd_0.71 & 5.33 & 5.64 & 5.54 & 5.49 & 0.05 & 0.05 & -0.41 \\
B_1d_0.71 & 5.33 & 5.64 & 5.54 & 5.49 & 0.05 & 0.05 & -0.41 \\
C_df_p_0.71 & 5.75 & 5.68 & 5.67 & 5.71 & -0.03 & 0.04 & 0.07 \\
C_df_1.00_inter & 5.21 & 5.48 & 5.42 & 5.34 & 0.08 & -0.02 & -0.33 \\
C_ld_1.00_inter & 5.21 & 5.48 & 5.42 & 5.34 & 0.08 & -0.02 & -0.33 \\
C_dd_0.71_inter & 5.08 & 5.37 & 5.27 & 5.15 & 0.11 & -0.02 & -0.39 \\
C_df_0.71_cold & 5.17 & 5.48 & 5.39 & 5.35 & 0.04 & 0.05 & -0.40 \\
C_ld_0.71_inter & 5.08 & 5.37 & 5.27 & 5.15 & 0.12 & -0.01 & -0.39 \\
C_1d_0.71_cold & 5.18 & 5.48 & 5.39 & 5.35 & 0.04 & 0.05 & -0.39 \\
C_hd_0.71_inter & 5.08 & 5.37 & 5.27 & 5.16 & 0.12 & -0.01 & -0.39 \\
C_ld_0.71_hot & 5.07 & 5.34 & 5.25 & 5.14 & 0.12 & -0.03 & -0.36 \\
C_df_0.71_hot & 5.07 & 5.34 & 5.25 & 5.14 & 0.12 & -0.03 & -0.36 \\
C_ld_0.60_inter & 5.15 & 5.42 & 5.33 & 5.33 & -0.01 & 0.10 & -0.35 \\
C_ddf0.60_inter & 5.15 & 5.42 & 5.33 & 5.33 & -0.01 & 0.10 & -0.35 \\
Stellar SED (C) & 5.38 & 5.28 & 5.28 & 5.33 & -0.05 & 0.05 & 0.10 \\
\hline
\end{tabular}

Notes. $m_{1}=(v-b)-(b-y) ; c_{1}=(u-v)-(v-b)$.

Supplement to the 2MASS All Sky Data Release and Extended Mission Products"7).

\subsubsection{Radio emission: $\mathrm{HI}-21 \mathrm{~cm}$ and rotational $\mathrm{CO}$ lines}

The $21 \mathrm{~cm}$ line of neutral hydrogen $(\mathrm{H} \mathrm{I}-21 \mathrm{~cm})$ and molecular rotational lines, such as $\mathrm{CO}_{1-0} \lambda=2.6806 \mathrm{~mm}$ and $\mathrm{CO}_{2-1} \lambda=$ $1.3004 \mathrm{~mm}$, may help to map the cold (neutral) outer regions of the outflow. Although the $21 \mathrm{~cm}$ line may be completely blended with ISM lines, the high speed of the ejected material (with terminal velocities up to $\approx 900 \mathrm{~km} \mathrm{~s}^{-1}$ ) may help to distinguish between the circumstellar and ISM contributions. In our models,

\footnotetext{
7 http://www.ipac.caltech.edu/2mass/releases/allsky/ doc/explsup.html, 2014-03-05, Sect. VI.4.a.
} 
Table 4. Magnitudes in SDSS bands and corresponding colour indices.

\begin{tabular}{lccccccc}
\hline \hline Model & $u^{\prime}$ & $g^{\prime}$ & $r^{\prime}$ & $i^{\prime}$ & $z^{\prime}$ & $u^{\prime}-g^{\prime}$ & $g^{\prime}-r^{\prime}$ \\
\hline A_df_0.71 & 4.83 & 5.06 & 5.23 & 5.40 & 5.86 & -0.22 & -0.17 \\
A_ld_0.71 & 4.84 & 5.06 & 5.23 & 5.40 & 5.86 & -0.22 & -0.17 \\
B_df_0.71 & 5.09 & 5.36 & 5.51 & 5.68 & 6.15 & -0.27 & -0.15 \\
B_ld_0.71 & 5.09 & 5.36 & 5.51 & 5.68 & 6.15 & -0.27 & -0.15 \\
C_df_p_0.71 & 5.43 & 5.51 & 5.81 & 6.04 & 6.23 & -0.08 & -0.30 \\
C_df_1.00_inter & 4.96 & 5.22 & 5.25 & 5.44 & 5.71 & -0.26 & -0.03 \\
C_ld_1.00_inter & 4.96 & 5.22 & 5.25 & 5.44 & 5.71 & -0.26 & -0.03 \\
C_df_0.71_inter & 4.84 & 5.08 & 5.24 & 5.44 & 5.89 & -0.25 & -0.16 \\
C_df_0.71_cold & 4.93 & 5.22 & 5.39 & 5.57 & 6.02 & -0.28 & -0.18 \\
C_ld_0.71_inter & 4.84 & 5.08 & 5.24 & 5.44 & 5.89 & -0.25 & -0.16 \\
C_ld_0.71_cold & 4.94 & 5.22 & 5.39 & 5.57 & 6.02 & -0.28 & -0.17 \\
C_hd_0.71_inter & 4.84 & 5.08 & 5.24 & 5.44 & 5.89 & -0.25 & -0.16 \\
C_ld_0.71_hot & 4.82 & 5.05 & 5.15 & 5.42 & 5.87 & -0.23 & -0.10 \\
C_df_0.71_hot & 4.82 & 5.05 & 5.15 & 5.42 & 5.87 & -0.23 & -0.10 \\
C_ld_0.60_inter & 4.90 & 5.17 & 5.36 & 5.55 & 5.99 & -0.27 & -0.19 \\
C_df_0.60_inter & 4.90 & 5.17 & 5.36 & 5.55 & 6.00 & -0.27 & -0.19 \\
Stellar SED (C) & 5.30 & 5.33 & 5.68 & 5.97 & 6.21 & -0.03 & -0.35 \\
\hline
\end{tabular}

the $\mathrm{CO}$ abundance is very small and does not lead to any observable lines. The H I-21 cm line is also very weak, possibly because of the low densities far from the star in the neutral hydrogen region. The $\mathrm{HI}-21 \mathrm{~cm}$ line luminosity does not exceed a few $10^{20} \mathrm{erg} \mathrm{s}^{-1}$ for both the dust-free and dusty simulations, which corresponds to a few $\mu \mathrm{Jy}$ at a resolving power of 2000 and a distance of $300 \mathrm{pc}$. The outer part of the outflow is therefore probably not detectable in these radio bands.

\subsection{Models with strong ionisation}

In this section, we investigate the impact of the hotspot temperature and SED on the emergent SED, as well as on the formation of dust and emission lines. A hotspot SED corresponding to a higher temperature implies more ionising photons and thus larger abundances of species, such as Si IV, N v, or C IV. Figure 11 shows the [Si IV] doublet at $139.4 \mathrm{~nm}$ and $140.3 \mathrm{~nm}$. There is no difference between the incident and emerging SEDs for the model with $T_{\mathrm{hs}}=30000 \mathrm{~K}$ ("C_df_0.71_low"). With a hotter hotspot and star, emission lines from strongly ionised elements can form (although still only barely noticeable for $T_{\text {hs }}=35000 \mathrm{~K}$; model “C_df_0.71_inter").

The bottom panel of Fig. 6 shows the relative abundance of Si IV and Fe II for the models C_df_0.71_inter and C_df_0.71_hot. We find that Si IV is restricted to the Roche volume around the gainer star. We therefore expect a strong lineprofile variability due to the orbital motion, as well as eclipses by the donor and the gainer stars as observed in some W Ser systems (see Appendix C). Unfortunately, we cannot simulate these effects in our models, which do not resolve the inner binary system. Owing to the short cooling time scale close to the star (Fig. 5), only the material above the hotspot (within the cone of opening angle $\theta_{\text {flow }}$ in Fig. 1) may contain highly ionised species. The low hotspot-temperature models produce much less Si IV, but more Fe II than the high-temperature ones.

The observed presence of [Si IV] emission in the Algolrelated systems W Ser (Sect. 4.1 and Appendix C) does not necessarily imply systemic mass loss, however, because it probes the inner part of the nebula, which may still be gravitationally bound. However, if present, the variability of lines from such highly ionised species may help to understand the ejection mechanism (Guinan 1989). Indeed, since the ionised elements form close to the hot region (the ejection point in the hotspot

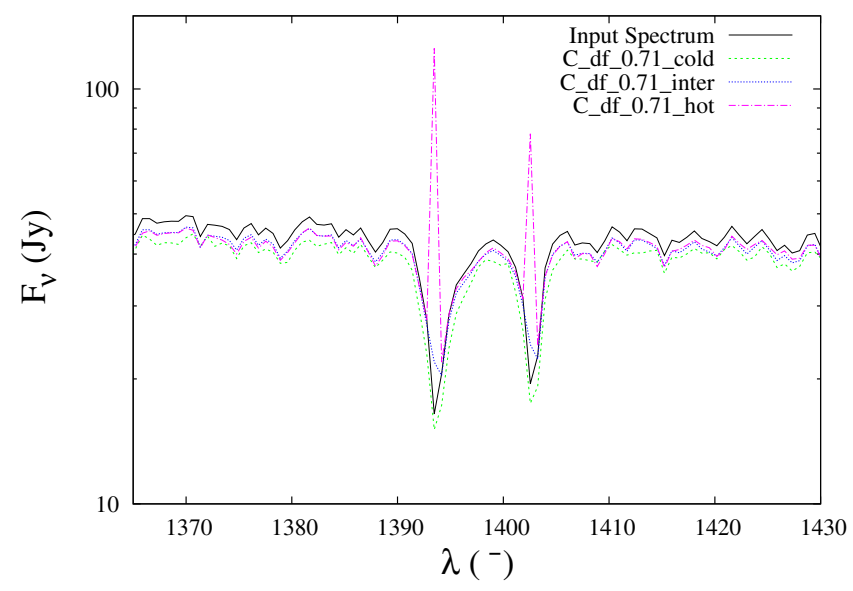

Fig. 11. [Si IV] 1394 and $1403 \AA$ doublet for three different configurations.

paradigm), we should be able to constrain the position of the UV source and check whether it is compatible with the location of the accretion point. On top of that, radial-velocity measurements may constrain the global motion of this material (expelled from the accretion region or revolving around the accreting star, etc.).

With increasing hotspot temperatures, only the outermost regions contain dust. Thus the following dichotomy is expected: (dust/weak ionisation) or (no dust/strongly ionised elements), which is supported by observations as discussed in Sect. 4.2.

\subsection{Parameter study: geometry - SKIRT simulations - dust-to-gas ratio}

In this section, we compare $1 \mathrm{D}$ results from CLOUDY with results from the $3 \mathrm{D}$ dust radiative-transfer code SKIRT. When the codes are applied to the same environment (as described by the stellar parameters, the opening angle of the stream, etc.), very good agreement between the 1D and 3D results is found (see Fig. 9: compare the dashed green line and dotted long-dashed cyan line). This agreement validates the results obtained with Cloudy and described so far.

As described in Sect. 2.5, we calculated models with different covering factors $f_{\mathrm{c}}$ representing different outflow geometries. Figure 7 shows the impact of the covering factor for three configurations: the spherical emission case $\left(f_{\mathrm{c}}=1\right)$ and two cases of a disc-like emission $\left(f_{\mathrm{c}}=0.71\right.$ and $\left.f_{\mathrm{c}}=0.6\right)$. For each covering factor, we recomputed the density profile according to Eq. (1) so that the total mass in the outflow is conserved. The UV/optical absorption increases with decreasing covering factor, because the densities increase, but the effect is modest. With the higher covering factors, the IR flux is significantly reduced because the dust condensation radius being larger, and less dust contributes to the infrared emission (Fig. 9).

Figure 12 shows a 2D map of the edge-on system at three different wavelengths $(12,25$, and $60 \mu \mathrm{m})$ produced with SKIRT using the same physical properties as in model C_ld_0.71_inter (same opening angle and dust-to-gas ratio $\Phi=2 \times 10^{-5}$; model C_SK_ld). We restrained our investigation to regions where the flux is close to the actual detection limits, set at $0.1 \mathrm{mJy} \operatorname{arcsec}^{-2}$ $(\approx 1 / 10$ of current observational thresholds in the mid- to nearIR for the VLT/VISIR instrument; Lagage et al. 2004). We do not expect a strong contribution from the cosmic infrared background (CIB) at the selected wavelengths. Schlegel et al. (1998) show that at $100 \mu \mathrm{m}$ (their lowest wavelength), the CIB 
R. Deschamps et al.: Non-conservative evolution in Algols: where is the matter?

Table 5. Magnitudes in 2MASS and WISE bands assuming the models are located $300 \mathrm{pc}$ away from the Sun.

\begin{tabular}{lcccccccccc}
\hline \hline Model & $J$ & $H$ & $K_{\mathrm{s}}$ & $W 1$ & $W 2$ & $W 3$ & $W 4$ & $F_{J} / F_{K_{\mathrm{s}}}$ & $F_{W 4} / F_{W 1}$ & $F_{W 3} / F_{W 1}$ \\
\hline A_df_0.71 & 5.24 & 5.46 & 5.23 & 5.31 & 5.23 & 5.01 & 4.70 & 2.39 & 0.05 & 0.14 \\
A_ld_0.71 & 5.23 & 5.46 & 5.23 & 5.31 & 5.23 & 4.19 & 3.22 & 2.41 & 0.19 & 0.29 \\
B_df_0.71 & 5.52 & 5.72 & 5.45 & 5.46 & 5.27 & 4.95 & 4.58 & 2.27 & 0.06 & 0.16 \\
B_ld_0.71 & 5.52 & 5.72 & 5.44 & 5.46 & 5.28 & 4.24 & 3.35 & 2.26 & 0.19 & 0.31 \\
C_df_p_0.71 & 5.67 & 5.60 & 5.51 & 5.43 & 5.35 & 4.93 & 4.58 & 2.09 & 0.06 & 0.16 \\
C_df_1.00_inter & 5.44 & 5.68 & 5.59 & 5.77 & 5.71 & 5.46 & 5.08 & 2.80 & 0.05 & 0.14 \\
C_ld_1.00_inter & 5.44 & 5.68 & 5.56 & 5.77 & 5.70 & 4.61 & 3.41 & 2.80 & 0.24 & 0.30 \\
C_df_0.71_inter & 5.39 & 5.63 & 5.44 & 5.51 & 5.41 & 5.11 & 4.71 & 2.54 & 0.06 & 0.15 \\
C_df_0.71_cold & 5.45 & 5.67 & 5.42 & 5.45 & 5.31 & 4.99 & 4.64 & 2.37 & 0.06 & 0.16 \\
C_ld_0.71_inter & 5.39 & 5.63 & 5.44 & 5.51 & 5.40 & 4.49 & 3.27 & 2.54 & 0.21 & 0.26 \\
C_ld_0.71_cold & 5.44 & 5.67 & 5.43 & 5.46 & 5.32 & 4.21 & 3.20 & 2.39 & 0.22 & 0.33 \\
C_hd_0.71_inter & 5.39 & 5.64 & 5.44 & 5.49 & 5.17 & -0.21 & -1.99 & 2.54 & 26.43 & 19.43 \\
C_ld_0.71_hot & 5.37 & 5.63 & 5.45 & 5.53 & 5.41 & 4.35 & 3.17 & 2.59 & 0.24 & 0.30 \\
C_df_0.71_hot & 5.37 & 5.63 & 5.45 & 5.53 & 5.42 & 5.14 & 4.74 & 2.60 & 0.06 & 0.15 \\
C_ld_0.60_inter & 5.38 & 5.59 & 5.31 & 5.34 & 5.21 & 4.20 & 3.12 & 2.27 & 0.21 & 0.29 \\
C_df_0.60_inter & 5.38 & 5.59 & 5.31 & 5.34 & 5.23 & 4.93 & 4.60 & 2.28 & 0.05 & 0.15 \\
Stellar SED (C) & 5.76 & 5.77 & 5.76 & 5.76 & 5.75 & 5.73 & 5.74 & 2.41 & 0.04 & 0.11 \\
\hline
\end{tabular}

Notes. $F_{J} / F_{K_{\mathrm{s}}}, F_{W 4} / F_{W 1}$ and $F_{W 3} / F_{W 1}$ are the flux ratios.

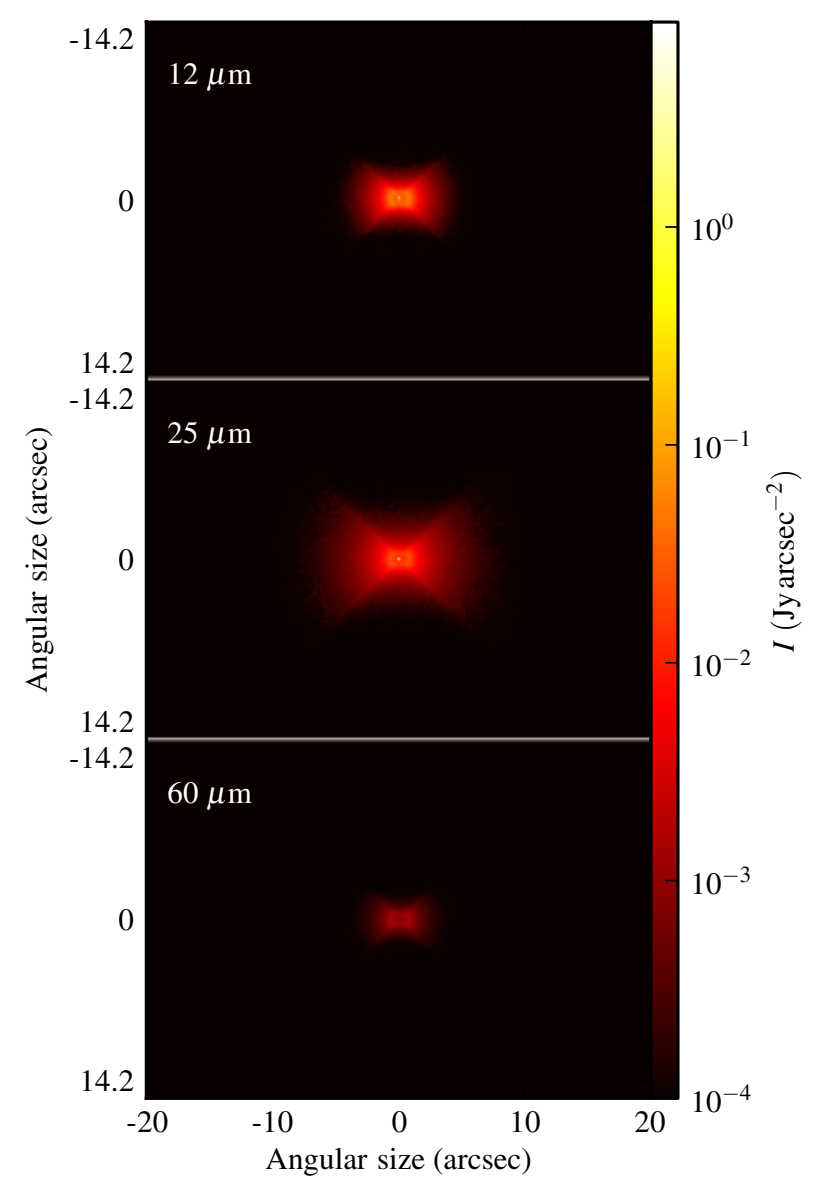

Fig. 12. SKIRT intensity map at $12 \mu \mathrm{m}$ (top), $25 \mu \mathrm{m}$ (middle), and $60 \mu \mathrm{m}$ (bottom) for $\Phi=2 \times 10^{-5}$ (model C_SK_ld). To ease the comparison, the same limits are used for the three bands for the " $x$ " and " $y$ " axes. The angular size and intensity are given for a system at $300 \mathrm{pc}$. The figure is zoomed on a box $12000 \times 8516 \mathrm{AU}^{2}$ wide.

value is only $\approx 3.3 \mathrm{mJy} \operatorname{arcsec}^{-2}$ in the galactic plane and not as close to the galactic pole and at shorter wavelengths. The speckle effect (coarseness observed especially far from the centre of the box) seen in Fig. 12 is due to the smaller number of photon packages sent from the outer regions compared to the inner regions, so it is numerical noise. The edges of the disc-like outflow are too cold to be seen at $12 \mu \mathrm{m}$, and only the longer wavelengths can probe these outer regions. However, the flux decreases at longer wavelengths and drops below the adopted detection limit. The "X" shape is due to the opening angle of the disc-like outflow, seen through the optically thin outer part. We recall that, in our models, there is no material outside the opening angle $\theta_{\text {flow }}$, whereas in a real system we expect a much smoother transition, with the highest density on the binary plane and a lower density at higher latitudes. Therefore, the " $\mathrm{X}$ " will be less marked. The inner hot region, where dust cannot form, has a diameter of $\approx 425$ AU corresponding to $\approx 1$ ". 4 (18 pixels) in Fig. 12. For comparison, the binary system has a separation of less than $1 \mathrm{AU}$ and is thus far from being resolved.

For the models (C_hd_0.71 and C_SK_hd) with a higher dust-to-gas ratio $\left(\Phi=4 \times 10^{-3}\right.$, typical of the ISM) and a covering factor $f_{\mathrm{c}}=0.71$, the $12 \mu \mathrm{m}$ peak rises to $\approx 60 \mathrm{Jy}$ in both the CLOUDY and the SKIRT simulations, as compared to $0.6 \mathrm{Jy}$ for the model C_ld_0.71_inter displayed in Figs. 9 and 12. A ratio of 100 between the $12 \mu \mathrm{m}$ fluxes of models differing by a factor of 200 in their dust-to-gas ratios may seem surprising when the dust shell is optically thin, as is the case here. However, one should first subtract the stellar contribution from the observed $12 \mu \mathrm{m}$ flux of model C_ld_0.71_inter and then take the ratio with the flux from model C_hd_0.71. The expected ratio of 200 is found then. Clearly, the uncertainty on the dust-to-gas ratio in the Algol environment has a strong impact on the present predictions.

\section{Comparison to observations}

\subsection{Stellar samples}

In this section, we present the samples of objects investigated for the presence of systemic mass loss as predicted by the models. The observable diagnostics extracted from the models and discussed in Sect. 3 are not typical of Algol systems, but instead share similarities with the properties of Algol-related systems, such as W Serpentis and $\beta$ Lyrae stars, $\mathrm{B}[\mathrm{e}]$ and Be stars, symbiotic Algols, and double periodic variables.

Algols are defined by the Simbad database ${ }^{8}$ as "detached eclipsing binaries showing the Algol paradox (the more massive

8 http://simbad.u-strasbg.fr/simbad/ 
component of the system is the less evolved)". However, many among the systems considered as Algols in the literature are actually semi-detached systems.. In the present study, we call "genuine Algols" those systems that are detached or semi-detached, showing the Algol paradox in the absence of UV emission lines (that are used as a property of W Ser systems as discussed next). We created our own sample of Algols based on the catalogues of Brancewicz \& Dworak (1980) containing 701 Algols ${ }^{9}$ and (Budding et al. 2004) containing $435 \mathrm{Algols}^{10}$. In these two catalogues, the classification "Algol" is based on the individual mass ratio.

W Serpentis and $\beta$ Lyrae are the prototypes of two subsets of Class-II ${ }^{11}$ Algol systems. The accreting star in these objects may be obscured by a thick accretion disc (see the review of Guinan 1989). They correspond to rapid mass-transfer systems most likely during case $\mathrm{B}$, or BB for $\beta$ Lyr itself (second phase of mass transfer following the case-B mass-transfer phase). The main property of W Ser systems is the presence of strong ultraviolet emission lines ([C IV], [C II], [He II], [N V], [Si IV], etc.; see Appendix C) seen at every phase (Plavec 1980, 1982, 1988, 1989; Guinan 1989). Our sample of W Ser is built on the catalogue of 31 peculiar emission-line Algols (PELAs; Gudel \& Elias 1996) after removing those systems from that list that show no evidence of these UV emission lines. The strong ultraviolet emission lines from highly ionised species observed in W Ser systems may be identified with those appearing in our model with the highest-temperature hotspot (C_df_0.71_hot). However, these spectral signatures cannot confirm the presence of material escaping the system since they form inside the Roche lobe of the star (see Sect. 3.3). Moreover, in W Ser systems, there are two contributions to the strongly ionised emission lines (Weiland et al. 1995, see Appendix C): the boundary layer between the disc and the star, responsible for the broad emission seen at every phase; and the hotspot, responsible for the highly phase-dependent narrow emission. There have also been reports of infrared emission that we will further investigate. Taranova \& Shenavrin (1997) report infrared excesses at 3.5 and $5 \mu \mathrm{m}$ possibly attributable to dusty material. Similarly, RY Sct possesses an extended dusty nebula (2000 AU wide; Grundstrom et al. 2007).

Be stars are rapidly rotating $\left(v>300 \mathrm{~km} \mathrm{~s}^{-1}\right)$, non-supergiant B stars showing strong Balmer emission lines or evidence of an ionised shell (see Rivinius et al. 2013 for a recent review). These stars form a dust-free, outwardly diffusing disc. In single Be stars, the formation mechanism of these decretion discs is related to the rapid rotation of the star, but some Be stars are interacting binaries, likely Algols. In this case, the formation of the disc and the star's rapid rotation could result from the RLOF accretion stream (Paczynski 1991; Colpi et al. 1991; Deschamps et al. 2013). Although there is no catalogue of truly Algol-like Be stars available yet, Harmanec (2001) has compiled an extensive list of Be stars in binary systems that may serve as an interesting starting point. In that list, we identified five Algol-like systems of particular interest because it will be apparent from our subsequent analysis: $\phi$ Per (Gies et al. 1998), SX Aur (Linnell et al. 1988), HR 2142 (Peters 1983; Waters et al. 1991),

\footnotetext{
9 http://cdsarc.u-strasbg.fr/viz-bin/Cat?II/150A

${ }^{10}$ http://cdsarc.u-strasbg.fr/viz-bin/Cat?]/A\%2bA/417/ 263

11 Rapid mass transfer systems with mass ratio reversed (Deschamps et al. 2013).
}

$\pi$ Aqr (Bjorkman et al. 2002) and CX Dra (Berdyugin \& Piirola 2002).

$\mathrm{B}[\mathrm{e}]$ stars are $\mathrm{B}$ stars that show strong forbidden emission lines, Balmer emission lines, and infrared excesses due to hot circumstellar dust (Lamers et al. 1998). However, this class contains several poorly understood systems and includes young and evolved, single, and binary stars. Therefore, different mechanisms at the origin of the gas surrounding $\mathrm{B}[\mathrm{e}]$ stars may be involved, among which some may be related to mass transfer across the B[e] binary systems (see, e.g., Dunstall et al. 2012). There is no specific catalogue of binary $\mathrm{B}[\mathrm{e}]$ stars.

Symbiotic Algols are a subtype of symbiotic stars. Although most symbiotic stars are assumed to host an accreting white dwarf (e.g., Mikołajewska 2007), it is not necessarily the case for all of them. For example, SS Lep (Blind et al. 2011) is a noneclipsing symbiotic, showing the Algol paradox, composed of a red-giant donor and a main-sequence accreting star. This system shows the presence of a dusty circumbinary disc or envelope (Jura et al. 2001). The main difference with standard Algols lies in the mass transfer scheme, which is believed to be wind Rochelobe overflow (WRLOF; Mohamed \& Podsiadlowski 2007) instead of standard RLOF. Owing to the long period of the system (260.3), mass accretion onto the gainer is supposed to occur via an accretion disc. Unfortunately, the properties of the hotspot on the edge of an accretion disc are badly constrained, which makes it difficult to predict systemic mass-loss rates and to compare with observational data. Moreover, it is not clear whether the hotspot mechanism can work with WRLOF, because of the lower mass-transfer rates.

Double periodic variables (DPVs) are interacting binaries exhibiting two closely linked periodic variations, the shortest one being the orbital period (Mennickent et al. 2003, 2005, 2008) and the second one of unknown nature but probably linked to the variations in the strength of the disc wind (Mennickent et al. 2012). They are considered as one specific evolutionary step for the more massive Algols. An interesting property of these objects is the surprising constancy of their orbital period, which is not expected in Algols undergoing RLOF mass transfer with a mass ratio different from 1 (Garrido et al. 2013). An explanation could be that systemic mass loss compensates for the period increase caused by mass transfer from a less massive star to its more massive companion. Mennickent et al. (2008) has already emphasised the need for further investigation of these systems. Some DPVs (the Galactic ones) already appear in our Algol or W Ser catalogues, but most of these objects have been found thanks to surveys in the Small Magellanic Cloud and are therefore too far away to allow any clear detection of an infrared excess. We therefore do not include them in this study.

\subsection{Constraint on the dust formation: IR diagnostics}

In this section, we search for systems that exhibit the infrared colours typical of dust emission. We select Class II and III objects (flaring and quiescent Algols, respectively, with a mass ratio already reversed, according to the nomenclature of Paper I), for which we expect most of the mass to have been ejected and dust to have formed. Class III objects should no longer show any signature of systemic mass loss, though, because of the short-lived outflow, but they were nevertheless considered for completeness. We extracted the IR data from the IRAS, 2MASS, and WISE catalogues. The use of the IRAS catalogues 
R. Deschamps et al.: Non-conservative evolution in Algols: where is the matter?

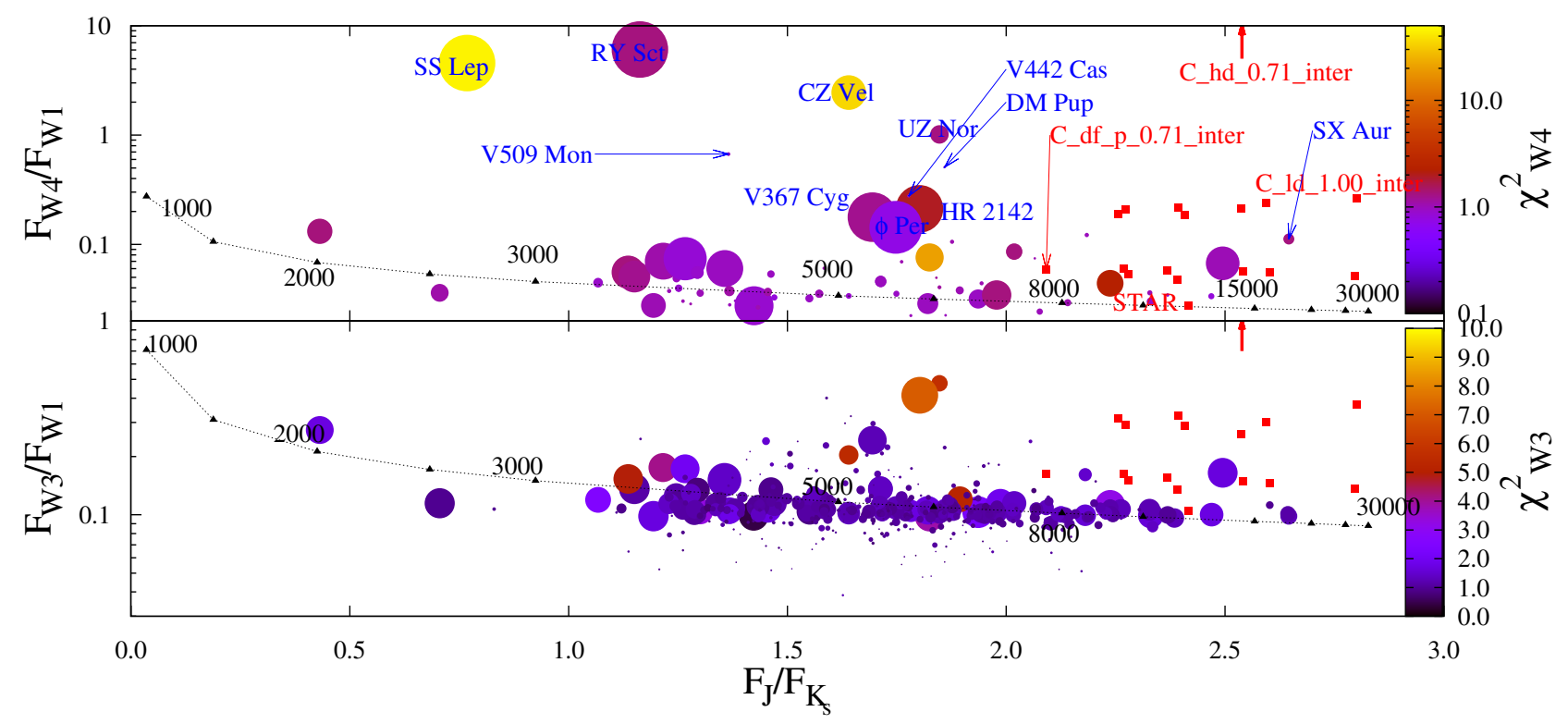

Fig. 13. Top panel: WISE $F_{W 4} / F_{W 1}$ against $2 \mathrm{MASS} \mathrm{J} / \mathrm{K}_{\mathrm{s}}$ flux ratios for Algols from the catalogue of Budding et al. (2004), and for W Ser from the list of Gudel \& Elias (1996). The symbiotic Algol SS Lep and a few Be and B[e] systems have been considered as well (as discussed in Sect. 4.1). The black filled triangles (linked by the dashed line) are black bodies of given temperatures (labels in Kelvin). The red filled squares represent the simulations (STAR: input spectrum). For clarity, only extreme models are labelled, and we refer to Table 5 for unlabelled models. The filled circles correspond to Algols with a $S / N>3.2$. The size of the circle is proportional to the $\mathrm{S} / \mathrm{N}$ for band $W 4$ (except for SS Lep which has a $\mathrm{S} / \mathrm{N}$ of 962 and for which we limit the size of the symbol), and the symbol colour relates to the $\chi^{2}$ for the same band. Bottom panel: same as top panel but for $F_{W 3} / F_{W 1}$ with $\mathrm{S} / \mathrm{N}$ and $\chi^{2}$ relative to $W 3$.

is motivated by their completeness in the 12 to $60 \mu \mathrm{m}$ range. On the other hand, the choice of the WISE and 2MASS databases has been motivated by their better detection limits as compared to IRAS (IRAS sensitivities: $0.5 \mathrm{Jy}$ at 12,25 , and $60 \mu \mathrm{m}$; WISE $5 \sigma$ sensitivities: $120,160,650$, and $2600 \mu \mathrm{Jy}$ at $3.3,4.7$, 12 , and $23 \mu \mathrm{m}$, respectively; 2MASS $10 \sigma$ at $J<15.0 \mathrm{mag}$, $\left.H<14.2 \mathrm{mag}, K_{\mathrm{s}}<13.5 \mathrm{mag}\right)$.

\subsubsection{IRAS data}

We selected the data from the IRAS (InfraRed All-sky Survey) point ${ }^{12}$ (Helou \& Walker 1988) and faint ${ }^{13}$ (Moshir \& et al. 1990) source catalogues. We retrieved 241 Algol entries with a matching IRAS source within a $2^{\prime}$ positional tolerance. We searched for IR excesses using the colour indices

$[i]-[j]=-2.5 \log \left(\frac{F_{i}}{F_{j}} \frac{F_{j, 0}}{F_{i, 0}}\right)$,

where $i$ and $j$ correspond to the IRAS bands at 12, 25, or $60 \mu \mathrm{m}$, and $F_{j, 0}$ and $F_{i, 0}$ are the zero-magnitude fluxes. After rejecting spurious detections (contaminated by a nearby infrared source), we did not find any systems presenting a deviation larger than 0.5 mag above the black-body line in the ([12]-[25], [12]-[60]) plane.

\subsubsection{WISE data}

$2 \mathrm{MASS}^{14}$ and $\mathrm{WISE}^{15}$ data are provided by Cutri et al. (2003) and Cutri et al. (2012), respectively. Figure 13 shows the flux ratios [WISE $F_{W 4}(22.1 \mu \mathrm{m}) / F_{W 1}(3.35 \mu \mathrm{m})$ ] and [WISE $F_{W 3}$ $\left.(11.6 \mu \mathrm{m}) / F_{W 1}(3.35 \mu \mathrm{m})\right]$ as functions of [2MASS $F_{J}$ $\left.(1.25 \mu \mathrm{m}) / F_{K_{\mathrm{s}}}(2.17 \mu \mathrm{m})\right]$ for 79 Algols having positional

\footnotetext{
12 http://cdsarc.u-strasbg.fr/viz-bin/Cat?II/125

${ }^{13}$ http://cdsarc.u-strasbg.fr/viz-bin/Cat?II/156A

${ }^{14}$ http://cdsarc.u-strasbg.fr/viz-bin/Cat?II/246

${ }^{15}$ http://cdsarc.u-strasbg.fr/viz-bin/Cat?II/311
}

matches with both 2MASS and WISE within less than $3^{\prime \prime}$. (31 objects have matches better than 0 ' $^{\prime} 12$ and 15 better than 0 .'06.) Spurious detections due to a diffraction spike from a close bright star, the persistence from a bright star scanned immediately before the target, and halo scattering from a close source or optical ghost have all been discarded. We retained systems having a signal-to-noise ratio $(\mathrm{S} / \mathrm{N})$ in band $W 4$ higher than 3.2. In addition, we note that the variability labels range from "not variable" (about 45 objects; this number depends on the band considered) to "high probability of being true variable" (34 objects). This variability observed by WISE could be attributed to an outflow, but it could also simply result from the eclipse of the donor by the gainer (in our model, the donor is three times brighter than the gainer in the $W 4$ band). A more precise analysis of variability with WISE data is beyond the scope of this study.

Figure 13 includes observed systems and our models. The circle colour codes the $\chi^{2}$ (which measures the deviation from the point-spread function-PSF; it is larger than 1 for extended sources) for bands $W 4$ and $W 3$. The circle size is proportional to the $\mathrm{S} / \mathrm{N}$ in bands $W 3$ and $W 4$. The $J$ and $K_{\mathrm{s}}$ bands are not affected by dust emission (see Fig. 9) and only depend on the stellar temperatures and on the covering factor. Since our simulations are based on one specific Algol system at three different stages of its evolution (see details in Table 1), the range in $F_{J} / F_{K_{\mathrm{s}}}$ values covered by the models is limited compared to observations. In the adopted Algol model system, the donor star dominates the spectrum at longer wavelengths beyond the $J$ band (Figs. 9 and 12).

On the other hand, $F_{W 4} / F_{W 1}$ directly traces the presence of IR emission from dust grains (Fig. 9), and $F_{W 3} / F_{W 1}$ probes the silicate emission around $12 \mu \mathrm{m}$. The models distribute along three horizontal sequences in the upper panel of Fig. 13, dust-free models corresponding to the lowest $F_{W 4} / F_{W 1}$ value (close to the stellar locus), followed by low-dust models at an intermediate $F_{W 4} / F_{W 1}$ value, and finally the high-dust model "C_hd_0.71_inter" falling above the limit of the figure. 
Table 6. 2 MASS and WISE magnitudes for the 13 systems deviating the most from the $F_{W 4} / F_{W 1}$ black-body law (see Fig. 13).

\begin{tabular}{|c|c|c|c|c|c|c|c|c|c|}
\hline Name & $\begin{array}{r}P_{\text {orb }} \\
\text { (d) } \\
q\end{array}$ & $\begin{array}{l}(\mathrm{Sp} .1) \\
{[\mathrm{Sp} .2]}\end{array}$ & $\begin{array}{c}J \\
\text { (mag) } \\
\varpi \text { (mas) }\end{array}$ & $\begin{array}{c}H \\
(\mathrm{mag})\end{array}$ & $\begin{array}{c}K_{\mathrm{s}} \\
(\mathrm{mag})\end{array}$ & $\begin{array}{c}W 1 \\
(\mathrm{mag}) \\
{\left[\mathrm{SNR}_{\mathrm{W} 1}\right]\left(\chi_{\mathrm{W} 1}^{2}\right)}\end{array}$ & $\begin{array}{c}W 2 \\
(\mathrm{mag}) \\
{\left[\mathrm{SNR}_{\mathrm{W} 2}\right]\left(\chi_{\mathrm{W} 2}^{2}\right)}\end{array}$ & $\begin{array}{c}W 3 \\
(\mathrm{mag}) \\
{\left[\mathrm{SNR}_{\mathrm{W} 3}\right]\left(\chi_{\mathrm{W} 3}^{2}\right)}\end{array}$ & $\begin{array}{c}W 4 \\
(\mathrm{mag}) \\
{\left[\mathrm{SNR}_{\mathrm{W} 4}\right]\left(\chi_{\mathrm{W} 4}^{2}\right)}\end{array}$ \\
\hline \multicolumn{10}{|c|}{ Algols } \\
\hline CZ Vel & $\begin{array}{r}5.1927^{(1)} \\
0.50^{(3)}\end{array}$ & $\begin{array}{c}(\mathrm{B} 3)^{(3)} \\
{[\mathrm{B} 6]}\end{array}$ & $\begin{array}{l}9.441 \\
\text { Unk. }\end{array}$ & 9.192 & 9.032 & $\begin{array}{c}8.562 \\
{[50](1.75)}\end{array}$ & $\begin{array}{c}8.485 \\
{[52.6](1.79)}\end{array}$ & $\begin{array}{c}7.814 \\
{[48.2](5.46)}\end{array}$ & $\begin{array}{c}3.669 \\
{[42.9](35.4)}\end{array}$ \\
\hline UZ Nor & $\begin{array}{r}3.1960^{(2)} \\
0.18^{(3)}\end{array}$ & $\begin{array}{l}(\mathrm{A} 1)^{(2)} \\
{[\mathrm{K} 0 \mathrm{IV}]}\end{array}$ & $\begin{array}{c}10.262 \\
\text { Unk. }\end{array}$ & 10.079 & 9.982 & $\begin{array}{c}9.873 \\
{[48.3](3.27)}\end{array}$ & $\begin{array}{c}9.817 \\
{[57.4](2.82)}\end{array}$ & $\begin{array}{c}8.202 \\
{[40.8](5.7)}\end{array}$ & $\begin{array}{c}5.937 \\
{[22.9](1.33)}\end{array}$ \\
\hline V509 Mon & $\begin{array}{r}4.9174^{(3)} \\
0.32^{(3)}\end{array}$ & $\begin{array}{l}(\mathrm{B} 7)^{(3)} \\
{[\mathrm{G} 4 \mathrm{IV}]}\end{array}$ & $\begin{array}{l}12.595 \\
\text { Unk. }\end{array}$ & 12.171 & 11.987 & $\begin{array}{c}11.868 \\
{[46.3](1.23)}\end{array}$ & $\begin{array}{c}11.905 \\
{[43.9](1.37)}\end{array}$ & $\begin{array}{c}11.515 \\
{[6.5](1.14)}\end{array}$ & $\begin{array}{c}8.38 \\
{[4.7](0.992)}\end{array}$ \\
\hline DM Pup & $\begin{array}{c}3.567^{(12)} \\
\text { Unk. }\end{array}$ & $\begin{array}{l}(\mathrm{B} 5)^{(3)} \\
{[\mathrm{A} 2.5]}\end{array}$ & $\begin{array}{c}12.761 \\
\text { Unk. }\end{array}$ & 12.569 & 12.488 & $\begin{array}{c}12.057 \\
{[46.1](1.38)}\end{array}$ & $\begin{array}{c}12.035 \\
{[42.6](1.61)}\end{array}$ & $\begin{array}{c}11.215 \\
{[9.6](1.00)}\end{array}$ & $\begin{array}{c}8.868 \\
{[3.2](1.09)}\end{array}$ \\
\hline V442 Cas & $\begin{array}{r}3.5922^{(3)} \\
0.18^{(3)}\end{array}$ & $\begin{array}{l}(\mathrm{A} 7)^{(3)} \\
{[\mathrm{K} 2 \mathrm{IV}]}\end{array}$ & $\begin{array}{c}11.729 \\
\text { Unk. }\end{array}$ & 11.516 & 11.407 & $\begin{array}{c}11.286 \\
{[49.6](1.36)}\end{array}$ & $\begin{array}{c}11.271 \\
{[52.2](1.42)}\end{array}$ & $\begin{array}{c}10.961 \\
{[14.9](0.995)}\end{array}$ & $\begin{array}{c}8.76 \\
{[4.2](0.994)}\end{array}$ \\
\hline DH Her & $\begin{array}{l}\text { Unk. } \\
\text { Unk. }\end{array}$ & $\begin{array}{l}(\mathrm{A} 5)^{(6)} \\
{[\mathrm{Unk} .]}\end{array}$ & $\begin{array}{l}10.054 \\
\text { Unk. }\end{array}$ & 9.997 & 9.956 & $\begin{array}{c}10.134 \\
{[47.9](5.90)} \\
\text { Lyr }\end{array}$ & $\begin{array}{c}10.168 \\
{[53.6](6.06)}\end{array}$ & $\begin{array}{c}10.189 \\
{[26.9](1.12)}\end{array}$ & $\begin{array}{c}8.449 \\
{[5.4](0.979)}\end{array}$ \\
\hline RY Sct & $\begin{array}{l}11.12^{(8)} \\
0.23^{(10)}\end{array}$ & $\begin{array}{c}(\text { O9.7Ibpe })^{(9)} \\
{[\text { O6.5 I] }}\end{array}$ & $\begin{array}{c}6.245 \\
1.59\end{array}$ & 5.854 & 5.463 & $\begin{array}{c}4.955 \\
{[14.2](12.1)} \\
\text { V Ser }\end{array}$ & $\begin{array}{c}3.895 \\
{[19.4](51.0)}\end{array}$ & $\begin{array}{c}0.014 \\
{[21.3](0.550)}\end{array}$ & $\begin{array}{c}-0.922 \\
{[83.96](2.21)}\end{array}$ \\
\hline V367 Cyg & $\begin{array}{r}18.6^{(13)} \\
0.825^{(13)}\end{array}$ & $\begin{array}{l}(\mathrm{A} 7 \mathrm{I})^{(3)} \\
{[\mathrm{Unk} .]}\end{array}$ & $\begin{array}{c}5.202 \\
1.74\end{array}$ & 5.006 & 4.828 & $\begin{array}{c}4.72 \\
{[13.2](0.434)}\end{array}$ & $\begin{array}{c}4.038 \\
{[20.9](2.34)}\end{array}$ & $\begin{array}{c}3.781 \\
{[70.9](1.27)}\end{array}$ & $\begin{array}{c}2.676 \\
{[31.4](1.24)}\end{array}$ \\
\hline W Ser & $\begin{array}{r}14.154^{(3)} \\
0.64^{(3)}\end{array}$ & $\begin{array}{c}(\mathrm{B} \text { emb. })^{(11)} \\
{[\mathrm{F} 5 \mathrm{III}]}\end{array}$ & $\begin{array}{l}7.416 \\
0.70\end{array}$ & 6.109 & $\begin{array}{r}5.558 \\
\text { Sym }\end{array}$ & $\begin{array}{c}5.106 \\
{[13.6](0.737)} \\
\text { otic Algol }\end{array}$ & $\begin{array}{c}4.560 \\
{[18.2](3.65)}\end{array}$ & $\begin{array}{c}4.038 \\
{[69](1.72)}\end{array}$ & $\begin{array}{c}3.379 \\
{[31.3](1.36)}\end{array}$ \\
\hline SS Lep & $\begin{array}{r}260.3^{(5)} \\
0.47^{(5)}\end{array}$ & $\begin{array}{c}(\mathrm{A} 1 \mathrm{~V})^{(5)} \\
{[\mathrm{M} 6 \mathrm{II}]}\end{array}$ & $\begin{array}{c}2.094 \\
3.59\end{array}$ & 2.105 & 1.671 & $\mathrm{Be} \begin{array}{c}3.455 \\
{[39](186)}\end{array}$ & $\begin{array}{c}1.536 \\
{[64.3](195)}\end{array}$ & $\begin{array}{c}-1.307 \\
{[3](0.0094)}\end{array}$ & $\begin{array}{c}-2.117 \\
{[962](45.2)}\end{array}$ \\
\hline HR 2142 & $\begin{array}{r}\sim 80^{(16)} \\
\text { Unk. }\end{array}$ & $\begin{array}{c}\text { (B2IVne) }^{(16)} \\
\text { [Unk.] }\end{array}$ & $\begin{array}{c}5.088 \\
2.48\end{array}$ & 5.068 & 4.781 & $\begin{array}{c}4.828 \\
{[12.1](15.4)}\end{array}$ & $\begin{array}{c}3.756 \\
{[16](25.5)}\end{array}$ & $\begin{array}{c}3.31 \\
{[91.1](7.12)}\end{array}$ & $\begin{array}{c}2.592 \\
{[58.9](1.96)}\end{array}$ \\
\hline$\pi \mathbf{A q r}$ & $\begin{array}{l}84.1^{(15)} \\
0.16^{(15)}\end{array}$ & $\begin{array}{l}(\mathrm{B} 1 \mathrm{Ve})^{(4)} \\
{[\mathrm{A}-\mathrm{F}]^{(15)}}\end{array}$ & $\begin{array}{c}5.305 \\
4.17\end{array}$ & 5.365 & 5.351 & $\begin{array}{c}4.573 \\
{[13.8](1.22)}\end{array}$ & $\begin{array}{c}3.767 \\
{[16](2.10)}\end{array}$ & $\begin{array}{c}4.056 \\
{[73.8](1.68)}\end{array}$ & $\begin{array}{c}3.58 \\
{[41.7](1.02)}\end{array}$ \\
\hline CX Dra & $\begin{array}{r}6.696^{(17)} \\
0.23^{(15)}\end{array}$ & $\begin{array}{c}(\mathrm{B} 2.5 \mathrm{Ve})^{(17)} \\
{[\mathrm{F} 5 \mathrm{III}]^{(17)}}\end{array}$ & $\begin{array}{c}5.870 \\
2.52\end{array}$ & 5.805 & 5.486 & $\begin{array}{c}5.457 \\
{[17.4](1.27)}\end{array}$ & $\begin{array}{c}5.140 \\
{[32.9](2.07)}\end{array}$ & $\begin{array}{c}5.128 \\
{[77.9](4.48)}\end{array}$ & $\begin{array}{c}4.854 \\
{[49](1.11)}\end{array}$ \\
\hline$\phi$ Per & $\begin{array}{r}127^{(14)} \\
0.158^{(3)}\end{array}$ & $\begin{array}{c}(\mathrm{B} 2 \mathrm{e})^{(14)} \\
{[\mathrm{sdO}]}\end{array}$ & $\begin{array}{c}4.049 \\
4.54\end{array}$ & 3.955 & 3.709 & $\begin{array}{c}3.68 \\
{[8.7](0.247)}\end{array}$ & $\begin{array}{c}2.774 \\
{[12.2](1.59)}\end{array}$ & $\begin{array}{c}2.639 \\
{[53.5](2.03)}\end{array}$ & $\begin{array}{c}1.869 \\
{[65.4](0.737)}\end{array}$ \\
\hline SX Aur & $\begin{array}{l}1.21^{(4)} \\
0.61^{(7)}\end{array}$ & $\begin{array}{l}(\mathrm{B} 2)^{(4)} \\
{[\mathrm{B} 5]}\end{array}$ & $\begin{array}{c}8.431 \\
1.39\end{array}$ & 8.471 & 8.541 & $\begin{array}{c}8.594 \\
{[47.9](2.97)}\end{array}$ & $\begin{array}{c}8.655 \\
{[55.6](3.53)}\end{array}$ & $\begin{array}{c}8.636 \\
{[42](1.29)}\end{array}$ & $\begin{array}{c}7.047 \\
{[13.6](1.34)}\end{array}$ \\
\hline
\end{tabular}

Notes. "Unk." refers to unknown values, "emb." to an embedded star where the spectral type is only roughly guessed. $q$ is the mass ratio defined as $q=M_{\text {donor }} / M_{\text {gainer. }}$.

References. (1) Zasche (2009); (2) Zasche (2011); (3) Budding et al. (2004) and references therein; (4) Linnell et al. (1988); (5) Blind et al. (2011); (6) Kreiner (2004); (7) Linnell et al. (1988); ${ }^{(8)}$ Kreiner (2004); ${ }^{(9)}$ Walborn (1982); (10) Grundstrom et al. (2007); ${ }^{(11)}$ Plavec (1989); ${ }^{(12)}$ Hegedus (1988); ${ }^{(13)}$ Zoła \& Ogłoza (2001); ${ }^{(14)}$ Hummel \& Štefl $(2001) ;{ }^{(15)}$ Bjorkman et al. $(2002) ;{ }^{(16)}$ Waters et al. (1991); (17) Berdyugin \& Piirola (2002).

Figure 13 reveals that several Algols and a few W Ser and $\beta$ Lyr in our sample have $F_{W 4} / F_{W 1}$ ratios falling above the black-body sequence, thus hinting at the presence of dust emission. The same holds true for the $F_{W 4} / F_{W 2}$ ratio, which has not been displayed, though, because the $W 2$ flux may reach (close to) saturation for bright systems. For four Algols (CZ Vel, UZ Nor, DM Pup and V509 Mon), one $\beta$ Lyr (RY Sct), and the symbiotic Algol SS Lep, $F_{W 4} / F_{W 1}$ is close to or larger than 1 , well above our model predictions for a dust-to-gas ratio of $\Phi=2 \times 10^{-5}$. The S/Ns of the WISE measurements for these systems are among the highest in our sample (see Table 6). The WISE and 2MASS data for these systems ${ }^{16}$ and others

\footnotetext{
${ }^{16}$ We thus do not confirm the conclusion of Taranova \& Shenavrin (1997), who seemingly found evidence of dust around the W Ser system RX Cas, though at shorter wavelengths (in the $L M$ bands at $3-5 \mu \mathrm{m}$ ). For this system, we found no significant infrared excesses from WISE $\left(F_{W 4} / F_{W 1}=0.056\right)$. Therefore, the excess found by Taranova \& Shenavrin (1997) in the $L M$ bands cannot come from dust.
}

with moderate excesses are collected in Table 6. Amongst these, not much is known (apart from their eclipsing light curve) for DM Pup, V509 Mon, CZ Vel, UZ Nor, V442 Cas, and DH Her, but given the interesting nature of all the other stars picked up by the $F_{W 4} / F_{W 1}$ criterion, a dedicated study of this short list of not well-studied stars will certainly pay off. RY Sct is a very interesting contact binary consisting of two seemingly O-type supergiants (but the $11 \mathrm{~d}$ orbital period makes it unlikely that the components are really as extended as supergiants are), with dust forming in an ionising radiation field and with a radiation-driven nebular wind with a kinematic age of $120 \mathrm{yr}$ (e.g., Smith et al. 2001).

Finally, three more systems have $F_{W 4} / F_{W 1}$ ratios close to the value expected for $\Phi=2 \times 10^{-5}$ : the Be systems V696 Mon (=HR 2142), $\phi$ Per, and SX Aur. SX Aur shows an extended shell in the W4 band as can be seen in Fig. 14. This is an interesting result in view of the controversy regarding the exact evolutionary status of that system: from the light curve analysis, Chambliss \& Leung (1979) obtained a semi-detached 

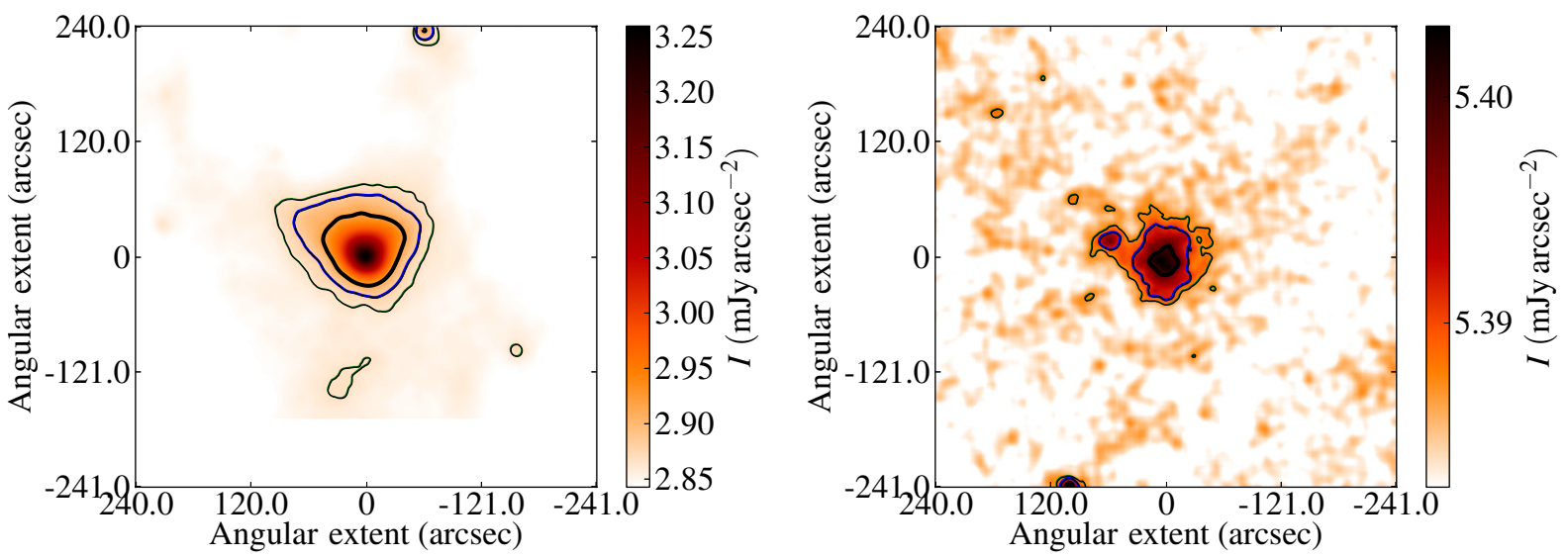

Fig. 14. WISE band-4 images for the genuine Algol CZ Vel (left) and the Be star SX Aur (right). The images are centred on the objects, with north up and east to the left. The flux scale goes from the background level to background plus 150 times the background scatter $\sigma$, and the first thin (green) contour line corresponds to the background level plus 3 times its fluctuation, the next intermediate (blue) contour curve to $5 \sigma$, and the inermost thick black contour line to $10 \sigma$.

configuration with the secondary filling its Roche lobe and endorse an earlier suggestion by Stothers (1973) that the system is undergoing case-A mass transfer and that mass reversal has occurred. In contrast, based on a new light curve analysis, Linnell et al. (1988) concluded that the system is made of two unevolved B stars, the primary being, however, on the verge of filling its Roche lobe. Our discovery of an extended infrared shell around SX Aur (Fig. 14) seems to suggest that some mass was lost from the system in the recent past, even though mass transfer is not currently occurring. This result confirms the interest of also considering Be stars when looking for evidence of systemic mass loss from Algols (see Sect. 4.1). Concerning HR 2142, it must be noted that the target lies close to a star-forming region (Monoceros R2 at 56'), and it is likely that the infrared emission does not originate in the binary system itself but rather in the rich sky background.

Amongst the systems with the highest $F_{W 4} / F_{W 1}$ ratios, we note that $\mathrm{CZ}$ Vel is the only genuine Algol system with an extended nebula detected by WISE (Fig. 14), as could be inferred by its high $\chi_{W 4}^{2}$. Its largest angular size is about $180^{\prime \prime}$. Unfortunately, the parallax of CZ Vel is unknown, as is therefore the linear size of the nebula. The nebula is very asymmetric and does not resemble our predictions (Fig. 12).

There is something else to notice from Fig. 13. Systems like W Ser or V930 Oph (having the lowest $F_{J} / F_{K_{\mathrm{s}}}=0.4$ and 0.71 , respectively) exhibit very low equivalent black-body temperatures $(T<3000 \mathrm{~K})$; in fact, they are well below the components' effective temperatures. These low temperatures thus indicate that the system light is, at those infrared wavelengths, dominated by a very cool object, possibly an accretion disc that obscures the gainer star, as in $\mathrm{W}$ Ser. The very short period $(P=1.4$; Malkov 1993) of V930 Oph does not support, however, the presence of an accretion disc; direct impact of the accreting stream is expected instead (Lubow \& Shu 1975). The low-temperature black body detected is therefore likely to originate in a substantial amount of cool dust far from the system, which is indicative of systemic mass loss.

Figure 13 shows that a typical ISM dust-to-gas ratio $(\Phi=$ $4 \times 10^{-3}$, model C_hd_0.71) produces $F_{W 4} / F_{W 1}$ ratios that are higher than those observed with WISE in Algols with an infrared excess. Thus, observations favour a lower dust-to-gas ratio in the stellar nebula than in the ISM. This conclusion is consistent with dust being destroyed by high-energy photons from both the B star and the hotspot. For the same reason, it is likely that so few genuine Algols exhibit infrared excesses caused by dust. Moreover, in the framework of the hotspot mechanism (Paper I), systemic mass loss occurs in the early stage of mass transfer. Since the circumstellar shell dissipates very quickly (within 300 years) after the cessation of the non-conservative evolution, this is another reason that infrared excesses are expected to be rare around genuine Algols.

Similarly, W Ser systems rarely show the signature of dust emission. This is compatible with our finding that the presence of highly ionised species (as seen in W Ser systems) is exclusive of the presence of dust (see Sect. 3.3).

\subsection{Constraint on Strömgren photometry and weakly ionised emission lines}

As mentioned in Sect. 3.2.1, the presence of a strong Balmer continuum in emission and the forest of metallic-line emission in the emergent spectrum are not typical of Algols. Through its $c_{1}$ index (Sect. 3.2.5 and Fig. 10), Strömgren photometry offers an easy way to check for the presence of Balmer emission continuum. Usually, as concluded before, IR excesses and emission lines or continua are mutually exclusive. There are three interesting exceptions, though: the $\beta$ Lyr system RY Sct, consisting of two stars with O-type spectra (Smith et al. 2001) and dust formation in the circumstellar nebula despite ionising radiation (Gehrz et al. 1995). We confirm the finding of Gehrz et al. (1995), since RY Sct is the system with the highest $F_{W 4} / F_{W 1}$ ratio (Table 6) and at the same time the smallest $c_{1,0}$ index (Table 7), which is indicative of a strong Balmer continuum in emission. The system clearly has all the features that indicate systematic mass loss. The Be stars $\phi$ Per and $\pi$ Aqr also present negative $c_{1,0}$, indicating a strong Balmer continuum. This property thus reveals once more the interest of Be binary systems in the search for Algollike systems with systemic mass loss.

In Table 7 , there are three other objects with negative $c_{1,0}$ values: the W Ser stars, AX Mon and V644 Mon, and the Be star HR 2142. All the other systems are located in a region of the $m_{1,0}-c_{1,0}$ diagram that is not typical of Balmer-continuum emission. On the contrary, the W Ser star V367 Cyg has large $c_{1,0}$ and deviates considerably from the main sequence locus. Young \& Snyder (1982) presented a dedicated study off $u v b y$ photometry of V367 Cyg (along with a few other W Ser systems) and found the same peculiar Strömgren colours as those reported here, which imply either excess flux longwards of 
Table 7. Strömgren $m_{1,0}$ and $c_{1,0}$ for our sample of W Ser and Be stars.

\begin{tabular}{|c|c|c|c|c|}
\hline Name & Type & $m_{1,0}(\mathrm{mag})$ & $c_{1,0}(\mathrm{mag})$ & Ref. \\
\hline RY Sct & $\beta$ Lyr & -0.0218 & -0.3002 & (4) \\
\hline \multirow{3}{*}{$\beta \mathrm{Lyr}$} & $\beta$ Lyr & 0.0697 & 0.32 & (11) \\
\hline & & 0.07006 & 0.3186 & (2) \\
\hline & & 0.06518 & 0.3278 & (6) \\
\hline \multirow[t]{4}{*}{ AU Mon } & W Ser & 0.05866 & 0.3186 & (1) \\
\hline & & 0.04928 & 0.2748 & (5) \\
\hline & & 0.0599 & 0.144 & (10) \\
\hline & & 0.06386 & 0.3976 & (7) \\
\hline KX And & W Ser & 0.01472 & 0.1662 & (7) \\
\hline \multirow[t]{3}{*}{ RZ Sct } & W Ser & -0.00166 & 0.1054 & (1) \\
\hline & & -0.01142 & 0.0768 & (9) \\
\hline & & 0.01804 & 0.1594 & (6) \\
\hline UX Mon & W Ser & 0.14302 & 0.3742 & (10) \\
\hline V356 Sgr & W Ser & 0.0435 & 0.527 & (9) \\
\hline \multirow[t]{4}{*}{ V367 Cyg } & W Ser & 0.03818 & 1.4508 & (1) \\
\hline & & 0.03744 & 1.4494 & (7) \\
\hline & & 0.0383 & 1.445 & (8) \\
\hline & & 0.03424 & 1.4944 & (6) \\
\hline \multirow[t]{3}{*}{ W Cru } & W Ser & 0.41384 & 0.4614 & (1) \\
\hline & & 0.41672 & 0.4352 & (13) \\
\hline & & 0.4062 & 0.538 & (9) \\
\hline W Ser & W Ser & 0.14036 & 0.3076 & (9) \\
\hline V395 Aur & $\mathrm{Be}$ & 0.15024 & 0.2954 & (6) \\
\hline V617 Aur & $\mathrm{Be}$ & 0.14018 & 0.2518 & (7) \\
\hline V644 Mon & $\mathrm{Be}$ & -0.01952 & -0.0202 & (10) \\
\hline V1914 Cyg & $\mathrm{Be}$ & 0.17682 & 0.1162 & (12) \\
\hline U CrB & $\mathrm{Be}$ & 0.07786 & 0.4606 & (6) \\
\hline AX Mon & $\mathrm{Be}$ & -0.02574 & -0.1174 & (6) \\
\hline$\phi$ Per & $\mathrm{Be}$ & 0.0336 & -0.095 & (4) \\
\hline CX Dra & $\mathrm{Be}$ & 0.06112 & 0.2262 & (14) \\
\hline \multirow[t]{3}{*}{$\pi \mathrm{Aqr}$} & $\mathrm{Be}$ & 0.00188 & -0.1892 & (1) \\
\hline & & -0.00296 & -0.2096 & (2) \\
\hline & & 0.0038 & -0.179 & (3) \\
\hline \multirow[t]{3}{*}{ HR 2142} & $\mathrm{Be}$ & 0.02558 & -0.0042 & (1) \\
\hline & & 0.0236 & 0.017 & (2) \\
\hline & & 0.02648 & -0.0152 & (3) \\
\hline
\end{tabular}

References. (1) Hauck \& Mermilliod (1998); (2) Grønbech \& Olsen (1976); ${ }^{(3)}$ Crawford et al. (1971); ${ }^{(4)}$ Westin (1982); ${ }^{(5)}$ Knude (1992); (6) Cameron (1966); (7) Olsen (1983); ( ${ }^{8)}$ Perry (1969); (9) Wolf \& Kern (1983); ${ }^{(10)}$ Kilkenny et al. (1985); ${ }^{(11)}$ Hauck \& Mermilliod (1980); (12) Olsen (1993); (13) Gray \& Olsen (1991); ${ }^{(14)}$ Crawford et al. (1971).

$400 \mathrm{~nm}$ or a strong absorption in the $u$ band, due for instance to improperly corrected interstellar absorption. The latter interpretation is made likely by V367 Cyg being a remote object in the Cygnus dust clouds. Therefore, Young \& Snyder (1982) conclude that "there is reason to question the reddening correction" in such a peculiar environment. Of course, one cannot firmly exclude an alternative reason related to the physics of the binary system, even though the $\left(m_{1,0}, c_{1,0}\right)$ colours of V367 Cyg are not typical of the other W Ser systems studied here and by Young \& Snyder (1982).

\section{Conclusions}

We performed photoionisation and radiative-transfer simulations of the material surrounding an Algol system that is losing mass via the hotspot mechanism. We calculated models with various configurations (different hotspot temperatures, with or without dust, different opening angles for the disc-like outflow), and found that the resulting IR excesses produced by dust in the outer parts of the outflow are consistent with WISE observations for a small number of Algols, especially the systems CZ Vel,
UZ Nor, and V509 Mon. We also reported infrared excess in the Be system SX Aur, in the $\beta$ Lyrae system RY Sct, and in the symbiotic Algol SS Lep (already known for the last two). As expected, most genuine Algols do not exhibit strong infrared excesses since the systemic mass loss occurred in an earlier phase, and the material has dissipated since. Our models also predict strong Balmer continua that are not observed in Algols but are visible in some Be systems.

The outflowing gas can significantly reduce the observed UV continuum-flux level. We suspect that this absorption disappears quickly after the end of the non-conservative phase owing to the high outflow speed of the line-driven wind and shows some phase dependency because the matter is launched from the hotspot. This variability may help to identify systems undergoing systemic mass loss due to the hotspot mechanism.

Our simulations indicate that the geometry of the outflow has an impact on the emergent SEDs, especially in the IR range for dusty models, where the fluxes decrease with increasing opening angle of the outflow.

In the SEDs computed from our models with a hotspot temperature $T_{\mathrm{hs}} \gtrsim 35000 \mathrm{~K}$, we find strong emission lines of highly ionised species such as Si IV that arise close to the stellar surface, inside the Roche lobe of the gainer star, and they are typical of W Ser systems. We emphasise the need for further observations of the extended family of Algols, which includes DPVs, symbiotic Algols, Be and $\mathrm{B}[\mathrm{e}]$ binaries, W Ser, and $\beta$ Lyr systems. These types of objects can be related to different steps in the evolution of Algols or to different initial configurations (masses, periods). The search for signatures of a non-conservative evolution should not focus specifically on Algols, since they are older Class III systems (Paper I), but more generally on Algolrelated systems where systemic mass loss can potentially occur. We also hope that this first study of the hotspot-outflow formation will motivate a more precise analysis of the stream/star or stream/disc interaction, preferably by using 3D models including radiation-hydrodynamics (see Bisikalo et al. 2000; Nazarenko \& Glazunova 2013, for $\beta$ Lyr).

Acknowledgements. R.D. acknowledges support from the Communauté française de Belgique - Actions de Recherche Concertées and benefits from a European Southern Observatory studentship. K.B. is an F.R.S.-FNRS postdoctoral fellow and acknowledges support from contract 2.4501.12. L.S. is an FNRS Researcher. P.C. is supported by the CHARM network, part of the phase VII Interuniversity Attraction Pole (IAP) programme organised by the Belgian Science Policy Office (BELSPO). This research has made use of the VizieR catalogue access tool and SIMBAD database, CDS, Strasbourg, France. This publication makes use of data products from the Wide-field Infrared Survey Explorer, which is a joint project of the University of California, Los Angeles, and the Jet Propulsion Laboratory/California Institute of Technology, funded by the National Aeronautics and Space Administration, as well as of data products from the Two Micron All Sky Survey, which is a joint project of the University of Massachusetts and the Infrared Processing and Analysis Center, funded by the National Aeronautics and Space Administration and the National Science Foundation.

\section{References}

Ak, H., Chadima, P., Harmanec, P., et al. 2007, A\&A, 463, 233

Allende Prieto, C., Lambert, D. L., \& Asplund, M. 2001, ApJ, 556, L63 Allende Prieto, C., Lambert, D. L., \& Asplund, M. 2002, ApJ, 573, L137 Baes, M., Davies, J. I., Dejonghe, H., et al. 2003, MNRAS, 343, 1081

Baes, M., Verstappen, J., De Looze, I., et al. 2011, ApJS, 196, 22 Banks, T., Sullivan, D. J., \& Budding, E. 1990, Ap\&SS, 173, 77 Barniske, A., Oskinova, L. M., \& Hamann, W.-R. 2008, A\&A, 486, 971 Berdyugin, A., \& Piirola, V. 2002, A\&A, 394, 181

Bessell, M. S. 2011, PASP, 123, 1442

Bisikalo, D. V., Harmanec, P., Boyarchuk, A. A., Kuznetsov, O. A., \& Hadrava, P. 2000, A\&A, 353, 1009 
Bjorkman, K. S., Miroshnichenko, A. S., McDavid, D., \& Pogrosheva, T. M. 2002, ApJ, 573, 812

Blind, N., Boffin, H. M. J., Berger, J.-P., et al. 2011, A\&A, 536, A55

Brancewicz, H. K., \& Dworak, T. Z. 1980, Acta Astron., 30, 501

Budding, E., Erdem, A., Çiçek, C., et al. 2004, A\&A, 417, 263

Cameron, R. C. 1966, Georgetown Obs. Monogram, 21, 0

Camps, P., \& Baes, M. 2015, Astron. Comput., 9, 20

Castor, J. I., Abbott, D. C., \& Klein, R. I. 1975, ApJ, 195, 157

Chambliss, C. R., \& Leung, K.-C. 1979, ApJ, 228, 828

Chaubey, U. S. 1979, Ap\&SS, 64, 177

Colpi, M., Nannurelli, M., \& Calvani, M. 1991, MNRAS, 253, 55

Crawford, J. A. 1955, ApJ, 121, 71

Crawford, D. L., Barnes, J. V., \& Golson, J. C. 1971, AJ, 76, 1058

Cutri, R. M., Skrutskie, M. F., van Dyk, S., et al. 2003, VizieR Online Data Catalog: II/246

Cutri, R. M., Skrutskie, M. F., van Dyk, S., et al. 2012, VizieR Online Data Catalog: II/311

Deschamps, R., Siess, L., Davis, P. J., \& Jorissen, A. 2013, A\&A, 557, A40

Dunstall, P. R., Fraser, M., Clark, J. S., et al. 2012, A\&A, 542, A50

ESA 1997, The HIPPARCOS and TYCHO catalogues, Astrometric and photometric star catalogues derived from the ESA HIPPARcos Space Astrometry Mission, ESA SP, 1200

Ferland, G. J., Porter, R. L., van Hoof, P. A. M., et al. 2013, Rev. Mex. Astron. Astrofis., 49, 137

Fukugita, M., Ichikawa, T., Gunn, J. E., et al. 1996, AJ, 111, 1748

Garrido, H. E., Mennickent, R. E., Djurašević, G., et al. 2013, MNRAS, 428, 1594

Gehrz, R. D., Hayward, T. L., Houck, J. R., et al. 1995, ApJ, 439, 417

Gies, D. R., Bagnuolo, Jr., W. G., Ferrara, E. C., et al. 1998, ApJ, 493, 440

Giuricin, G., Mardirossian, F., \& Mezzetti, M. 1983, ApJS, 52, 35

Goodricke, J. 1783, Roy. Soc. London Phil. Trans. Ser. I, 73, 474

Gray, R. O., \& Olsen, E. H. 1991, A\&AS, 87, 541

Grevesse, N., \& Sauval, A. J. 1998, Space Sci. Rev., 85, 161

Grønbech, B., \& Olsen, E. H. 1976, A\&AS, 25, 213

Grundstrom, E. D., Gies, D. R., Hillwig, T. C., et al. 2007, ApJ, 667, 505

Gudel, M., \& Elias, II, N. M. 1996, in Radio Emission from the Stars and the Sun, eds. A. R. Taylor, \& J. M. Paredes, ASP Conf. Ser., 93, 312

Guinan, E. F. 1989, Space Sci. Rev., 50, 35

Harmanec, P. 2001, Pub. Astron. Inst. Czechoslovak Academy of Sciences, 89,

Harmanec, P. 2002, Astron. Nachr., 323, 87

Hauck, B., \& Mermilliod, M. 1980, A\&AS, 40, 1

Hauck, B., \& Mermilliod, M. 1998, A\&AS, 129, 431

Hegedus, T. 1988, Bull. Inf. Centre de Données Stellaires, 35, 15

Helou, G., \& Walker, D. W. 1988, Infrared astronomical satellite (IRAS) catalogs and atlases, Vol. 7: The small scale structure catalog

Holweger, H. 2001, in Joint SOHO/ACE workshop, Solar and Galactic Composition, ed. R. F. Wimmer-Schweingruber, AIP Conf. Ser., 598, 23

Hummel, W., \& Štefl, S. 2001, A\&A, 368, 471

Ignace, R., Oskinova, L. M., Waldron, W. L., Hoffman, J. L., \& Hamann, W.-R. 2008, A\&A, 477, L37

Jura, M., Webb, R. A., \& Kahane, C. 2001, ApJ, 550, L71

Kenyon, S. J. 1986, The symbiotic stars (Cambridge, New York: Cambridge University Press)

Kilkenny, D., Whittet, D. C. B., Davies, J. K., et al. 1985, South African Astron. Obs. Circ., 9, 55

Knude, J. 1992, A\&AS, 92, 841

Kopal, Z. 1955, Ann. Astrophys., 18, 379

Kreiner, J. M. 2004, Acta Astron., 54, 207

Lagage, P. O., Pel, J. W., Authier, M., et al. 2004, The Messenger, 117, 12

Lamers, H. J. G. L. M., Zickgraf, F.-J., de Winter, D., Houziaux, L., \& Zorec, J. 1998, A\&A, 340, 117

Lanz, T., \& Hubeny, I. 2007, ApJS, 169, 83

Laor, A., \& Draine, B. T. 1993, ApJ, 402, 441

Linnell, A. P., Peters, G. J., \& Polidan, R. S. 1988, ApJ, 327, 265

Lomax, J. R., \& Hoffman, J. L. 2011, Bull. Soc. Roy. Sci. Liège, 80, 689

Lubow, S. H., \& Shu, F. H. 1975, ApJ, 198, 383

Maeder, A. 2009, Physics, Formation and Evolution of Rotating Stars, Astronomy and Astrophysics Library (Berlin, Heidelberg: Springer)

Malkov, O. Y. 1993, Bull. Inf. Centre de Données Stellaires, 42, 27
Manfroid, J., Sterken, C., Bruch, A., et al. 1991, A\&AS, 87, 481

Martin, P. G., \& Rouleau, F. 1991, in Extreme Ultraviolet Astronomy, eds. R. F. Malina, \& S. Bowyer, 341

Massevitch, A., \& Yungelson, L. 1975, Mem. Soc. Astron. It., 46, 217

Mathis, J. S., Rumpl, W., \& Nordsieck, K. H. 1977, ApJ, 217, 425

Mennickent, R. E., Pietrzyński, G., Diaz, M., \& Gieren, W. 2003, A\&A, 399, L47

Mennickent, R. E., Cidale, L., Díaz, M., et al. 2005, MNRAS, 357, 1219

Mennickent, R. E., Kołaczkowski, Z., Michalska, G., et al. 2008, MNRAS, 389, 1605

Mennickent, R. E., Kołaczkowski, Z., Djurasevic, G., et al. 2012, MNRAS, 427, 607

Mezzetti, M., Giuricin, G., \& Mardirossian, F. 1980, A\&A, 83, 217

Mikołajewska, J. 2007, Balt. Astron., 16, 1

Mohamed, S., \& Podsiadlowski, P. 2007, in 15th European Workshop on White Dwarfs, eds. R. Napiwotzki, \& M. R. Burleigh, ASP Conf. Ser., 372, 397

Moro, D., \& Munari, U. 2000, A\&AS, 147, 361

Moshir, M., et al. 1990, in IRAS Faint Source Catalogue, version 2.0

Mukherjee, J., Peters, G. J., \& Wilson, R. E. 1996, MNRAS, 283, 613

Nazarenko, V. V., \& Glazunova, L. V. 2013, Astron. Rep., 57, 294

Nelson, C. A., \& Eggleton, P. P. 2001, ApJ, 552, 664

Olsen, E. H. 1983, A\&AS, 54, 55

Olsen, E. H. 1993, A\&AS, 102, 89

Paczynski, B. 1991, ApJ, 370, 597

Perry, C. L. 1969, AJ, 74, 705

Peters, G. J. 1983, PASP, 95, 311

Piirola, V., Berdyugin, A., Mikkola, S., \& Coyne, G. V. 2005, ApJ, 632, 576

Plavec, M. J. 1980, Highlights of Astronomy, 5, 831

Plavec, M. J. 1982, in NASA Conf. Pub. 2338, ed. Y. Kondo, 526

Plavec, M. J. 1988, ESA SP, 281, 221

Plavec, M. J. 1989, Space Sci. Rev., 50, 95

Pringle, J. E., \& Wade, R. A. 1985, Interacting binary stars, Cambridge Astrophysics Series (Cambridge: Cambridge University Press)

Refsdal, S., Roth, M. L., \& Weigert, A. 1974, A\&A, 36, 113

Richards, M. T., \& Albright, G. E. 1999, ApJS, 123, 537

Rivinius, T., Carciofi, A. C., \& Martayan, C. 2013, A\&ARv, 21, 69

Sarna, M. J. 1993, MNRAS, 262, 534

Schlegel, D. J., Finkbeiner, D. P., \& Davis, M. 1998, ApJ, 500, 525

Siess, L., Izzard, R. G., Davis, P. J., \& Deschamps, R. 2013, A\&A, 550, A100

Smith, N., Gehrz, R. D., \& Goss, W. M. 2001, AJ, 122, 2700

Soydugan, F., Frasca, A., Soydugan, E., et al. 2007, MNRAS, 379, 1533

Stothers, R. 1973, PASP, 85, 363

Strömgren, B. 1966, ARA\&A, 4, 433

Sytov, A. Y., Kaigorodov, P. V., Bisikalo, D. V., Kuznetsov, O. A., \& Boyarchuk, A. A. 2007, Astron. Rep., 51, 836

Taranova, O. G., \& Shenavrin, V. I. 1997, Astron. Lett., 23, 698

Tokunaga, A. T., \& Vacca, W. D. 2005, PASP, 117, 421

Tout, C. A., \& Pringle, J. E. 1992, MNRAS, 256, 269

Umana, G., Maxted, P. F. L., Trigilio, C., et al. 2000, A\&A, 358, 229

Umana, G., Leone, F., \& Trigilio, C. 2002, A\&A, 391, 609

van Hoof, P. A. M., Weingartner, J. C., Martin, P. G., Volk, K., \& Ferland, G. J. 2004, MNRAS, 350, 1330

van Leeuwen, F. 2007, A\&A, 474, 653

van Rensbergen, W., De Greve, J. P., De Loore, C., \& Mennekens, N. 2008, A\&A, 487, 1129

van Rensbergen, W., De Greve, J. P., Mennekens, N., Jansen, K., \& De Loore, C. 2010, A\&A, 510, A13

van Rensbergen, W., de Greve, J. P., Mennekens, N., Jansen, K., \& de Loore, C. 2011, A\&A, 528, A16

Verner, E. M., Verner, D. A., Korista, K. T., et al. 1999, ApJS, 120, 101

Walborn, N. R. 1982, AJ, 87, 1300

Waters, L. B. F. M., Cote, J., \& Pols, O. R. 1991, A\&A, 250, 437

Weiland, J. L., Shore, S. N., Beaver, E. A., Lyons, R. W., \& Rosenblatt, E. I. 1995, ApJ, 447, 401

Westin, T. N. G. 1982, A\&AS, 49, 561

Wolf, G. W., \& Kern, J. T. 1983, ApJS, 52, 429

Young, A., \& Snyder, J. A. 1982, ApJ, 262, 269

Zasche, P. 2009, New Astron., 14, 129

Zasche, P. 2011, New Astron., 16, 157

Zoła, S., \& Ogłoza, W. 2001, A\&A, 368, 932 


\section{Appendix A: Estimation of the opening angle of the outflow}

The geometry of the escaping stream may be complex, forming a disc-like geometry, and possibly creating a spiral-like structure close to the star (see Sect. 2.2). The geometrical thickness of the disc structure is determined by the opening angle of the outflow. Since the outflow is driven by the radiative flux of momentum $\boldsymbol{p}_{v}$, we can estimate the geometrical thickness of the outflow by using the dependence of $\boldsymbol{p}_{v}$ on the direction given by $\theta_{n}$ (angle measured with respect to the direction perpendicular to the hotspot surface; see Fig. A.1).

The total momentum flux, which is normal to an element of surface $\mathrm{d} \mathcal{A}$ that is parallel to the hotspot, is given by (Fig. A.1)

$\boldsymbol{p}_{v}\left(\theta_{\mathrm{n}}=0\right)=\frac{I_{v}}{c} \overline{\boldsymbol{n}}_{\mathrm{h}} \int_{\text {hemisphere }} \cos ^{2}(\theta) \mathrm{d} \Omega$,

where $I_{v}$ is the specific intensity (assumed to be isotropic) originating in the hotspot, $\overline{\boldsymbol{n}}_{\mathrm{h}}$ is the unit vector normal to the hotspot, $c$ is the speed of light, and $\mathrm{d} \Omega=\sin \theta \mathrm{d} \theta \mathrm{d} \phi$.

To calculate the momentum flux towards an arbitrary angle $\theta_{\mathrm{n}}<\pi / 2$, we introduce the reference frame $(\widehat{x}, \widehat{y}, \widehat{z})$, where the $\widehat{x}$ axis lies in the plane of the hotspot, the $\widehat{y}$ axis lies in the plane $\mathrm{d} \mathcal{A}$, and $\widehat{z}$ is collinear with $\overline{\boldsymbol{n}}_{\mathrm{d} \mathcal{A}}$, the normal to $\mathrm{d} \mathcal{A}$. We note that $\theta$ is measured with respect to $\overline{\boldsymbol{n}}_{\mathrm{d} \mathcal{A}}$. The general expression of Eq. (A.1) for arbitrary angles $\theta_{\mathrm{n}}$ reads

$\boldsymbol{p}_{v}\left(\theta_{\mathrm{n}}\right)=\frac{I_{v}}{c} \overline{\boldsymbol{n}}_{\mathrm{d} \mathcal{A}} \int_{\text {hemisphere }} \cos \theta|\cos \theta| \Theta\left(\overline{\boldsymbol{n}} \cdot \overline{\boldsymbol{n}}_{\mathrm{h}}\right) \mathrm{d} \Omega$,

where $\overline{\boldsymbol{n}}$ is the unit vector pointing in the direction $(\theta, \phi)$. The absolute value in the second factor of the integrand ensures that radiation traversing the surface $\mathrm{d} \mathcal{A}$ from above adds a negative contribution to the total momentum flux. The Heavyside step function $\Theta\left(\overline{\boldsymbol{n}} \cdot \overline{\boldsymbol{n}}_{\mathrm{h}}\right)$ accounts for the fact that the hotspot only radiates upwards.

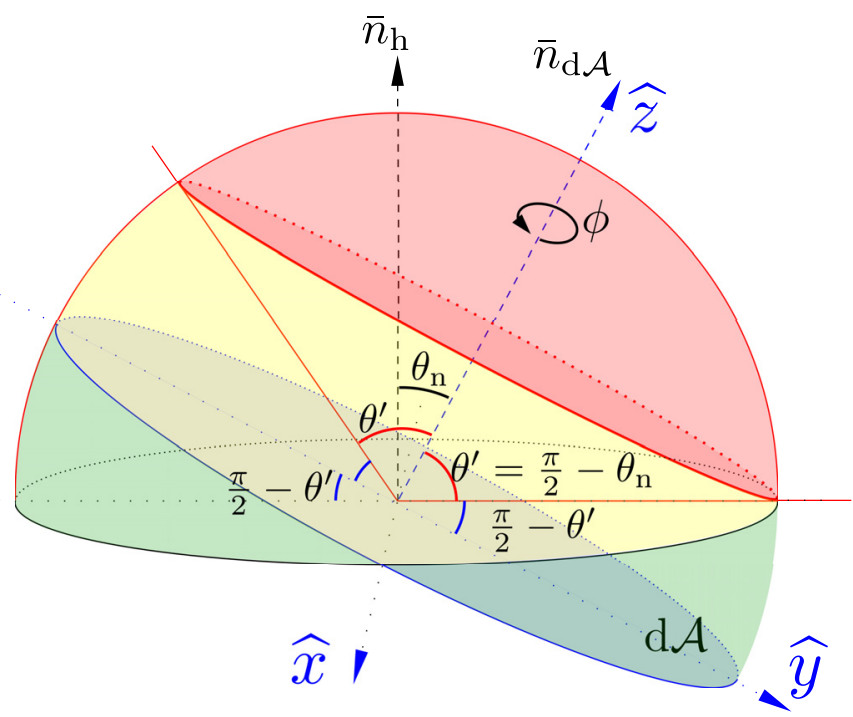

Fig. A.1. Geometry of the hotspot emission. $\overline{\boldsymbol{n}}_{\mathrm{h}}$ is the normal to the surface of the hotspot and $\overline{\boldsymbol{n}}_{\mathrm{d} \mathcal{A}}$ is the normal of the surface $\mathrm{d} \mathcal{A}$, which forms an angle $\theta_{\mathrm{n}}$ with $\overline{\boldsymbol{n}}_{\mathrm{h}}$. $\theta_{\text {flow }} / 2$ (see text) is equal to $\theta_{\mathrm{n}}$ when the momentum flux integrated in azimuthal angle between 0 and $\theta_{\mathrm{n}}$ equals $68 \%$ of the momentum flux integrated over the total hemisphere (between 0 and $\frac{\pi}{2}$ ).
For a fixed angle $\theta_{\mathrm{n}}$, the vector $\overline{\boldsymbol{n}}_{\mathrm{h}}$ normal to the hotspot has coordinates $\left(0,-\sin \theta_{\mathrm{n}}, \cos \theta_{\mathrm{n}}\right)$, so that an evaluation of the dot product gives the limits of integration in $\phi$ :

$$
\begin{aligned}
\phi_{\lim , 1} & = \begin{cases}\arcsin \left[1 /\left(\tan \theta \tan \theta_{\mathrm{n}}\right)\right] & \theta>\pi / 2-\theta_{\mathrm{n}} \\
0 & \text { otherwise }\end{cases} \\
\phi_{\lim , 2} & = \begin{cases}\pi-\arcsin \left[1 /\left(\tan \theta \tan \theta_{\mathrm{n}}\right)\right] & \theta>\pi / 2-\theta_{\mathrm{n}} \\
2 \pi & \text { otherwise }\end{cases}
\end{aligned}
$$

where the latter expression for $\phi_{\lim , 2}$ results from the symmetry of the problem (the angle $\phi$ is 0 above the $\widehat{x}$ axis). With these limits, Eq. (A.1) can be expressed as

$$
\begin{aligned}
\boldsymbol{p}_{v}\left(\theta_{\mathrm{n}}\right)= & \frac{I_{v}}{c} \overline{\boldsymbol{n}}_{\mathrm{d} \mathcal{A}}\left(\int_{0}^{2 \pi} \mathrm{d} \phi \int_{0}^{\pi / 2} \mathrm{~d} \theta \cos ^{2} \theta \sin \theta\right. \\
& \left.-2 \int_{\pi / 2-\theta_{\mathrm{n}}}^{\pi / 2} \mathrm{~d} \theta \cos ^{2} \theta \sin \theta \int_{\phi_{\mathrm{lim}, 1}}^{\phi_{\mathrm{lim}, 2}} \mathrm{~d} \phi\right) \\
= & \frac{I_{v}}{c} \overline{\boldsymbol{n}}_{\mathrm{d} \mathcal{A}}\left(\frac{2 \pi}{3}-2 \int_{\pi / 2-\theta_{\mathrm{n}}}^{\pi / 2} \mathrm{~d} \theta \cos ^{2} \theta \sin \theta \int_{\phi_{\mathrm{lim}, 1}}^{\phi_{\mathrm{lim}, 2}} \mathrm{~d} \phi\right),
\end{aligned}
$$

where the first integral (Eq. (A.5)) corresponds to the hemisphere over the surface element $\mathrm{d} \mathcal{A}$ in Fig. A.1, and the second integral (Eq. (A.6)) corresponds to the two green parts. The first of the two green parts is subtracted because it lies below the hotspot surface and does not contribute, and the second one makes a negative contribution to the momentum flux because it is below the surface element $\mathrm{d} \mathcal{A}$. These two regions are identical by symmetry, and they allow us to use the same limits of integration in $\phi$ and $\theta$.

Our estimate of the opening angle $\theta_{\text {flow }}$ of the outflow is based on the integral of the momentum flux $\boldsymbol{p}_{v}$ over $\theta_{\mathrm{n}}$. We define it as the angle that corresponds to a fraction of 0.68 (arbitrarily set; standard $1 \sigma$ as for a Gaussian distribution) of the total integral, i.e.,

$$
\int_{0}^{\theta_{\text {flow }} / 2} \boldsymbol{p}_{v}\left(\theta_{\mathrm{n}}\right) \mathrm{d} \theta_{\mathrm{n}}=0.68 \int_{0}^{\pi / 2} \boldsymbol{p}_{v}\left(\theta_{\mathrm{n}}\right) \mathrm{d} \theta_{\mathrm{n}} .
$$

This gives an opening angle of $\theta_{\text {flow }} \sim 90^{\circ}$. The "effective" solid angle in which the disc-like outflow spreads is given by:

$4 \pi f_{\mathrm{c}}=\int_{0}^{2 \pi} \mathrm{d} \phi \int_{\pi / 2-\theta_{\text {flow }} / 2}^{\pi / 2+\theta_{\text {flow }} / 2} \mathrm{~d} \theta \sin \theta=4 \pi \sin \left(\theta_{\text {flow }} / 2\right) \sim 2.84 \pi \mathrm{sr}$,

where $f_{\mathrm{c}}$ is the covering factor that is defined as the fraction of the spherical domain that is covered by gas (Sect. 2.1). This is different from the geometry adopted in Paper I, where the outflow was arbitrarily assumed to be confined in a quarter of a sphere (solid angle equal to $\pi$ ).

\section{Appendix B: Calculation of the effective flux}

In our simulations, the effective radiative flux $F_{\text {eff }}$ is given by

$F_{\text {eff }}=F_{\mathrm{g}}+\frac{\mathcal{A}_{\mathrm{hs}}}{\mathcal{A}_{\mathrm{eff}, \mathrm{hs}}} F_{\mathrm{hs}}$,

where $F_{\mathrm{g}}=L_{*} / \mathcal{A}_{*}$ and $F_{\mathrm{hs}}=L_{\mathrm{hs}} / \mathcal{A}_{\mathrm{hs}}$ are the gainer star and hotspot fluxes, respectively; $\mathcal{A}_{*}$ is the surface area of the star; and $\mathcal{A}_{\mathrm{hs}}$ is the surface area of the hotspot, i.e. the surface of a 
R. Deschamps et al.: Non-conservative evolution in Algols: where is the matter?

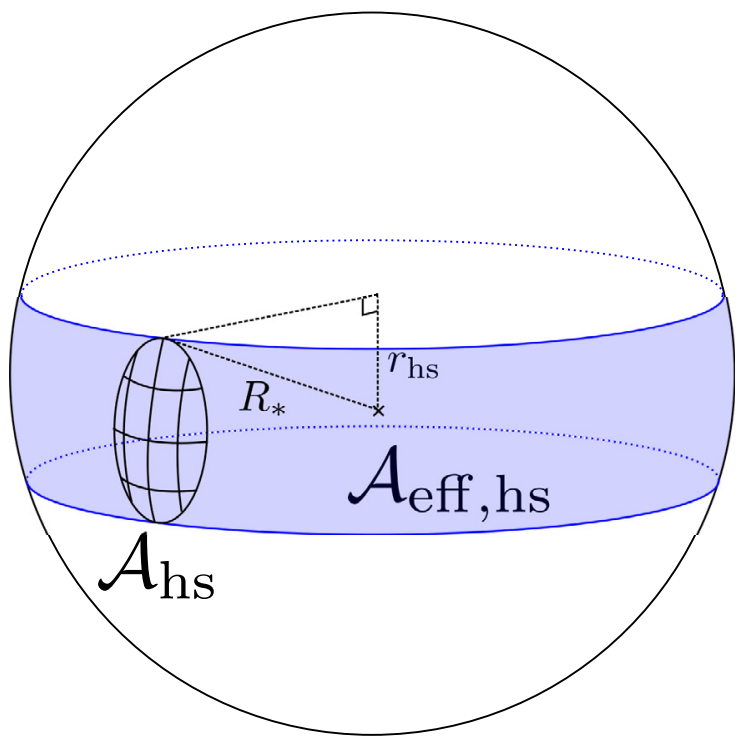

Fig. B.1. Representation of the effective surface of the hotspot over a full orbital period. See text for description.

calotte with the radius of the base equivalent to the radius of the impacting stream,

$\mathcal{A}_{\mathrm{hs}}=2 \pi R_{*}\left(R_{*}-\sqrt{R_{*}^{2}-r_{\mathrm{hs}}^{2}}\right)$,

where $R_{*}$ and $r_{\mathrm{hs}}$ are the star and hotspot radius. Here, $\mathcal{A}_{\mathrm{eff}}$,hs is the area of the strip at the equator of the star of height $2 r_{\mathrm{hs}}$ (Fig. B.1),

$\mathcal{A}_{\mathrm{eff}, \mathrm{hs}}=4 \pi R_{*}^{2}-2 \times 2 \pi R_{*}\left(R_{*}-r_{\mathrm{hs}}\right)$,

over which the hotspot flux is averaged. Therefore, the effective flux reads as

$F_{\mathrm{eff}}=F_{\mathrm{g}}+\frac{R_{*}}{2 r_{\mathrm{hs}}}\left[1-\sqrt{1-\left(\frac{r_{\mathrm{hs}}}{R_{*}}\right)^{2}}\right] F_{\mathrm{hs}}$.

The net flux from the hotspot is thus reduced by the factor

$\gamma=\frac{R_{*}}{2 r_{\mathrm{hs}}}\left[1-\sqrt{1-\left(\frac{r_{\mathrm{hs}}}{R_{*}}\right)^{2}}\right]$.

In our simulations, the source of radiation emits isotropically, so that the total effective luminosity will be

$L_{\mathrm{eff}}=L_{\mathrm{g}}+\mathcal{A}_{*} F_{\mathrm{eff}, \mathrm{hs}}=L_{\mathrm{g}}+\frac{R_{*}}{r_{\mathrm{hs}}} L_{\mathrm{hs}}$,

where $F_{\mathrm{eff}, \mathrm{hs}}=\left(\mathcal{A}_{\mathrm{hs}} / \mathcal{A}_{\mathrm{eff}, \mathrm{hs}}\right) F_{\mathrm{hs}}$.

\section{Appendix C: UV continuum and line variability in W Ser}

W Ser systems are known to show strong line variability in the UV (especially for highly ionised species; see the review by Plavec 1982). In this appendix, we investigate the observed UV spectra for the prototypical system W Ser in the search for variations in the continuum as presented in Sect. 3.2. We also demonstrate the variability of [Si IV] lines for this object.

Figure C.1 shows low-dispersion, large aperture spectra of W Ser in the UV as observed by the International Ultraviolet

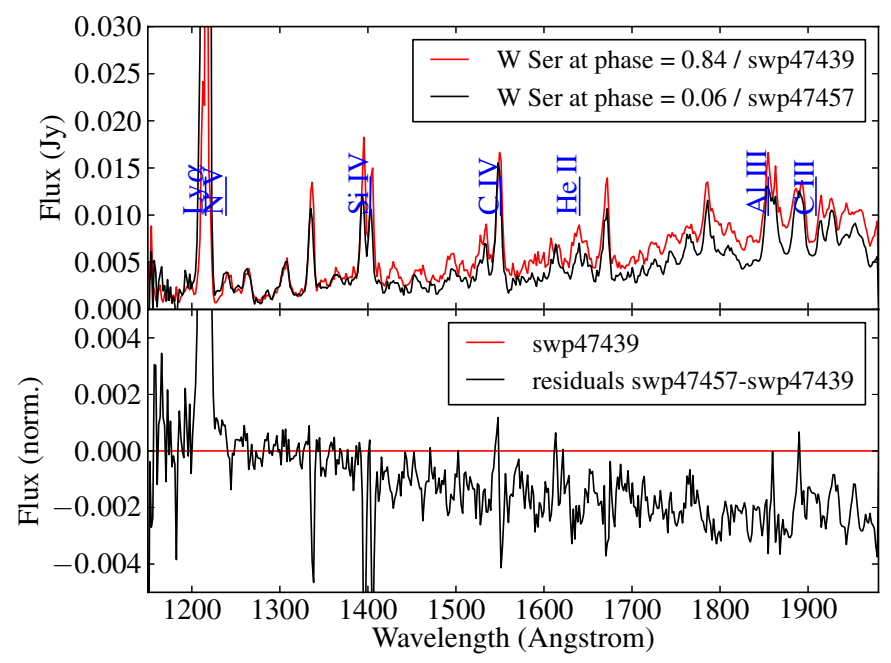

Fig. C.1. Top: continuum in the $U V$ band for W Ser observed with IUE. Black: SWP47439 observation made on 1993-04-07 (20:02:16) at phase $\Phi=0.84$; red: SWP47457 observation made on 1993-04-10 (21:40:17) at phase $\Phi=0.06$. Bottom: difference of the two spectra.

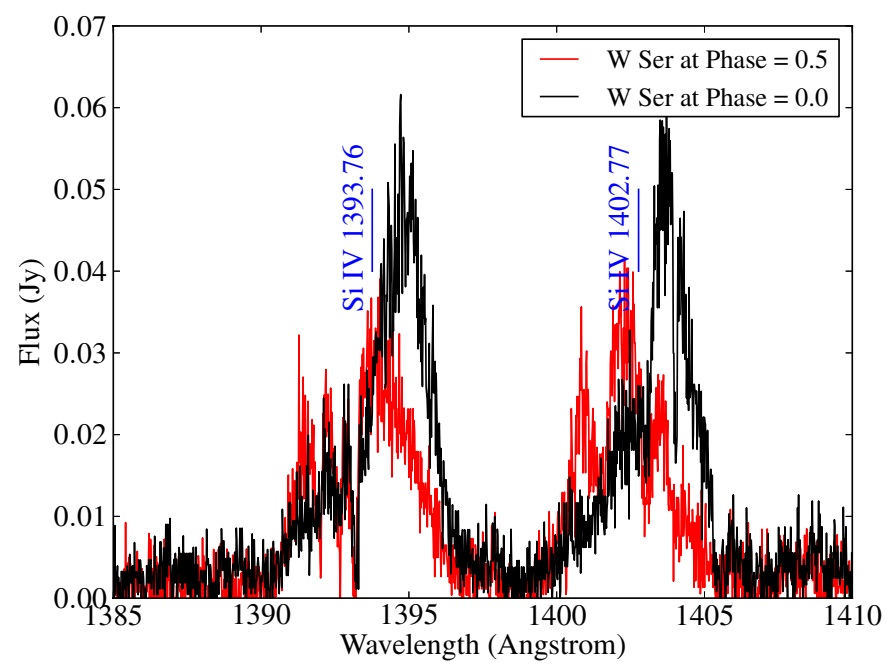

Fig. C.2. HST/GHRS spectra of the [SiIV] 1393.76 and $1402.77 \AA$ lines of W Ser at two distinct phases (black: z0lu5108t observation made on 1991-07-12 (05:32:25) at phase $\Phi=0.00$, primary eclipse; red: z0lu0308t observation made on 1991-07-19 (03:29:13) at phase $\Phi=0.5$, secondary eclipse).

Explorer (IUE) at two distinct phases. (These phases are estimated thanks to the improved ephemeris of Piirola et al. 2005.) The two spectra have been taken only three days apart, thus limiting the effect of intrinsic variability. The red spectrum is taken between the secondary and the primary eclipses at phase 0.8 and the black one at phase 0.1 during the primary eclipse. We note that at phase 0.1 , the absorption in the longer wavelength region of the spectra $(\lambda>1400 \AA)$ and the Lyman $\alpha$ emission are stronger than during the secondary eclipse, possibly indicating a hot ionised region.

Figure C. 2 presents the profiles of the [Si IV] 1393.76 and $1402.77 \AA$ lines as observed with HST/GHRS for W Ser at two different phases: primary eclipse $(\Phi=0.0)$, and secondary eclipse $(\Phi=0.5)$. The same ephemeris has been used. We clearly see an excess of flux in the blue wing at phase $\Phi=0.5$. The total extent of the wings of both lines reaches $600 \mathrm{~km} \mathrm{~s}^{-1}$, which is comparable to the escape velocity at the surface of 
the star $\left(643 \mathrm{~km} \mathrm{~s}^{-1}\right.$ considering a main sequence gainer star of $1.51 M_{\odot}$ according to Budding et al. 2004, and following a mass-radius relation for main-sequence stars of the form $R \propto M^{0.8}$ ) and with the outflow terminal velocity in our model $\left(850 \mathrm{~km} \mathrm{~s}^{-1}\right)$. In their attempt to model this line variability, Weiland et al. (1995) found two components, a broad one likely due to the boundary layer of an accretion disc, and a narrow one possibly due to the formation of a hotspot. Further investigation, especially at different phases, may shed new light on the origin of the [Si IV] emission.

\section{Appendix D: Chemical composition of the outflow}

Table D.1 presents the chemical composition of the donor star atmosphere that will be transferred by RLOF onto the accretor's surface and eventually ejected by the hotspot.

Table D.1. Chemical composition of the outflowing material for the three models computed.

\begin{tabular}{ccccc}
\hline \hline Element $(i)$ & $\begin{array}{c}M_{i} / M_{\text {tot }} \\
\text { (Model A) }\end{array}$ & $\begin{array}{c}M_{i} / M_{\text {tot }} \\
\text { (Model B ) }\end{array}$ & $\begin{array}{c}M_{i} / M_{\text {tot }} \\
\text { (Model C) }\end{array}$ & $\begin{array}{c}M_{i} / M_{\text {tot }} \\
\text { (Solar ref.) }\end{array}$ \\
\hline $\mathrm{H}$ & $6.9941 \times 10^{-1}$ & $6.9902 \times 10^{-1}$ & $6.9810 \times 10^{-1}$ & $7.3732 \times 10^{-1}$ \\
$\mathrm{He}$ & $2.8059 \times 10^{-1}$ & $2.8074 \times 10^{-1}$ & $2.8134 \times 10^{-1}$ & $2.4917 \times 10^{-1}$ \\
$\mathrm{Li}$ & $6.7290 \times 10^{-18}$ & $3.1061 \times 10^{-18}$ & $1.0826 \times 10^{-18}$ & $1.0358 \times 10^{-8}$ \\
$\mathrm{C}$ & $3.4675 \times 10^{-3}$ & $2.2462 \times 10^{-3}$ & $1.0944 \times 10^{-4}$ & $2.1526 \times 10^{-3}$ \\
$\mathrm{~N}$ & $1.0632 \times 10^{-3}$ & $2.5270 \times 10^{-3}$ & $4.9937 \times 10^{-3}$ & $8.7194 \times 10^{-4}$ \\
$\mathrm{O}$ & $9.6519 \times 10^{-3}$ & $9.6486 \times 10^{-3}$ & $9.6333 \times 10^{-3}$ & $5.7348 \times 10^{-3}$ \\
$\mathrm{~F}$ & $5.6122 \times 10^{-7}$ & $5.6411 \times 10^{-7}$ & $5.6984 \times 10^{-7}$ & $4.1970 \times 10^{-7}$ \\
$\mathrm{Ne}$ & $1.9697 \times 10^{-3}$ & $1.9696 \times 10^{-3}$ & $1.9575 \times 10^{-3}$ & $1.4762 \times 10^{-3}$ \\
$\mathrm{Na}$ & $4.0004 \times 10^{-5}$ & $4.0130 \times 10^{-5}$ & $5.2729 \times 10^{-5}$ & $3.5989 \times 10^{-5}$ \\
$\mathrm{Mg}$ & $7.5221 \times 10^{-4}$ & $7.5221 \times 10^{-4}$ & $7.5220 \times 10^{-4}$ & $6.1694 \times 10^{-4}$ \\
$\mathrm{Al}$ & $6.4825 \times 10^{-5}$ & $6.4825 \times 10^{-5}$ & $6.4825 \times 10^{-5}$ & $5.8225 \times 10^{-5}$ \\
$\mathrm{Si}$ & $8.1136 \times 10^{-4}$ & $8.1136 \times 10^{-4}$ & $8.1136 \times 10^{-4}$ & $7.1290 \times 10^{-4}$ \\
$\mathrm{P}$ & $7.1102 \times 10^{-6}$ & $7.1102 \times 10^{-6}$ & $7.1102 \times 10^{-6}$ & $7.2504 \times 10^{-6}$ \\
$\mathrm{~S}$ & $4.3015 \times 10^{-4}$ & $4.3015 \times 10^{-4}$ & $4.3015 \times 10^{-4}$ & $4.3159 \times 10^{-4}$ \\
$\mathrm{Cl}$ & $2.3983 \times 10^{-6}$ & $2.3983 \times 10^{-6}$ & $2.3983 \times 10^{-6}$ & $4.9535 \times 10^{-6}$ \\
heavy & $1.7395 \times 10^{-3}$ & $1.7395 \times 10^{-3}$ & $1.7395 \times 10^{-3}$ & \\
\hline
\end{tabular}

Notes. Contributions from different isotopes (when available) are added up. For the CLOUDY simulations we use the thirty lightest elements. For those not appearing in the table we adopt solar abundances.

References. Solar abundances: Grevesse \& Sauval (1998), except N, Ne, Mg, Si, and Fe (Holweger 2001), O (Allende Prieto et al. 2001), and C (Allende Prieto et al. 2002). 